\title{
A Concise Synthesis of Lentiginosine Derivatives Using a Pyridinium Formation via the Mitsunobu Reaction
}

\author{
Rabah Azzouz, Corinne Fruit*, Laurent Bischoff* and Francis Marsais \\ Laboratoire de Chimie Organique Fine et Hétérocyclique, CNRS UMR 6014,Université de Rouen, IRCOF-INSA, \\ Université de Rouen, B.P. 08, 76131 Mont-Saint-Aignan Cedex FRANCE
}

\section{SUPPORTING INFORMATION $-{ }^{1} \mathrm{H} \&{ }^{13} \mathrm{C}$ NMR, MS}

General. Unless otherwise stated, reactions were performed under a nitrogen atmosphere using freshly distilled solvents. All reactions were monitored by thin-layer chromatography with Merck silica gel 60 F254 pre-coated aluminum plates $(0.25 \mathrm{~mm})$. Flash chromatography was performed with indicated solvents using silica gel (particle size 30-63 $\mu \mathrm{m}$ ) purchased from Merck. ${ }^{1} \mathrm{H}$ and ${ }^{13} \mathrm{C}$ NMR spectra were recorded on a Bruker Avance at $300 \mathrm{MHz}$ for ${ }^{1} \mathrm{H}$ and $75 \mathrm{MHz}$ for ${ }^{13} \mathrm{C}$. Chemical shifts are reported relative to TMS, calibrated with chloroform or deuterium oxide. Coupling constants $J$ are in $\mathrm{Hz}$ and are reported as d (doublet), t (triplet), q (quartet).

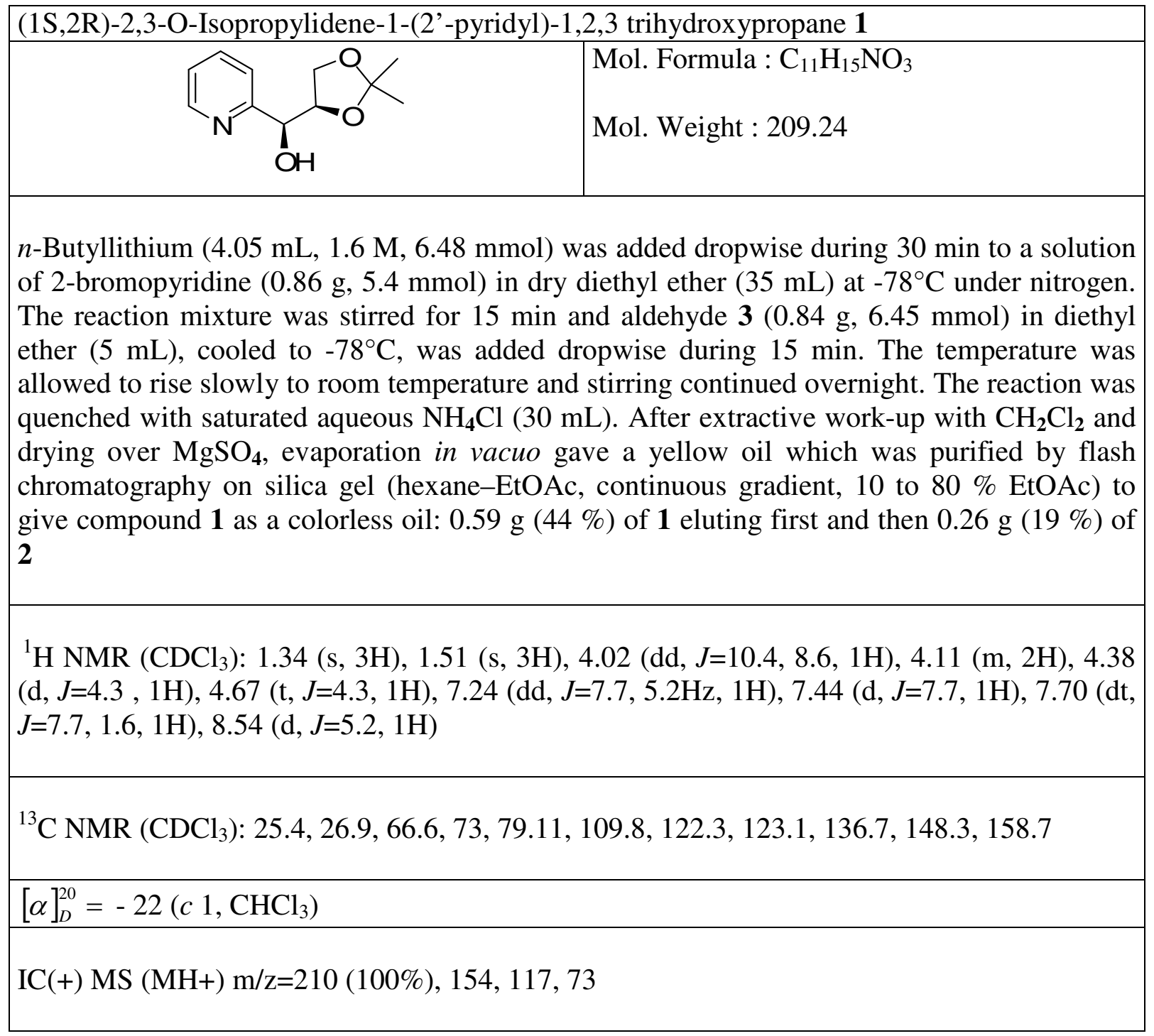




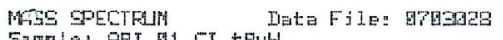

3I-MAR- 7 1日:5

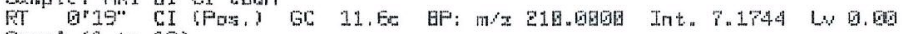

Suan! (1 to 12 )
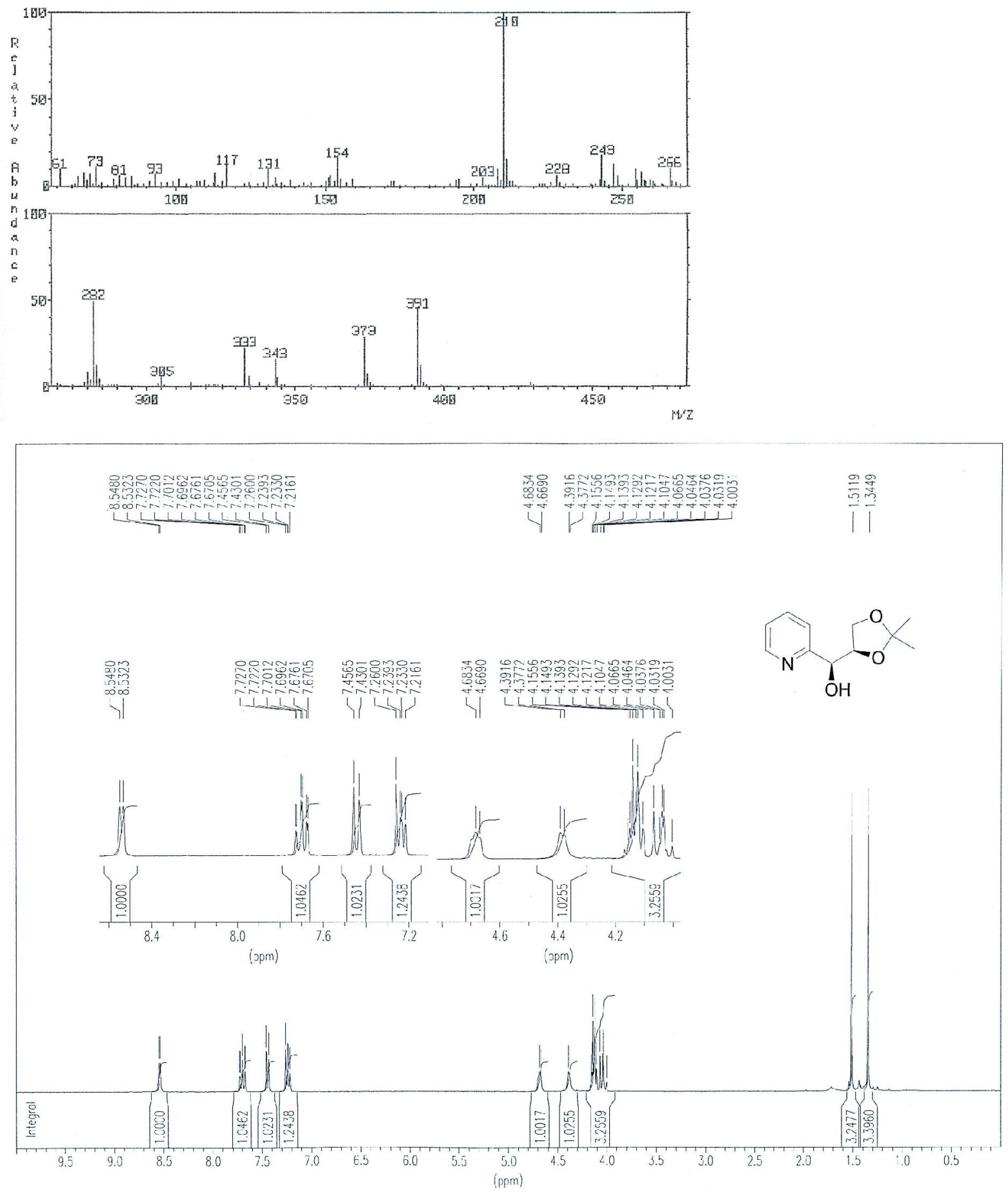


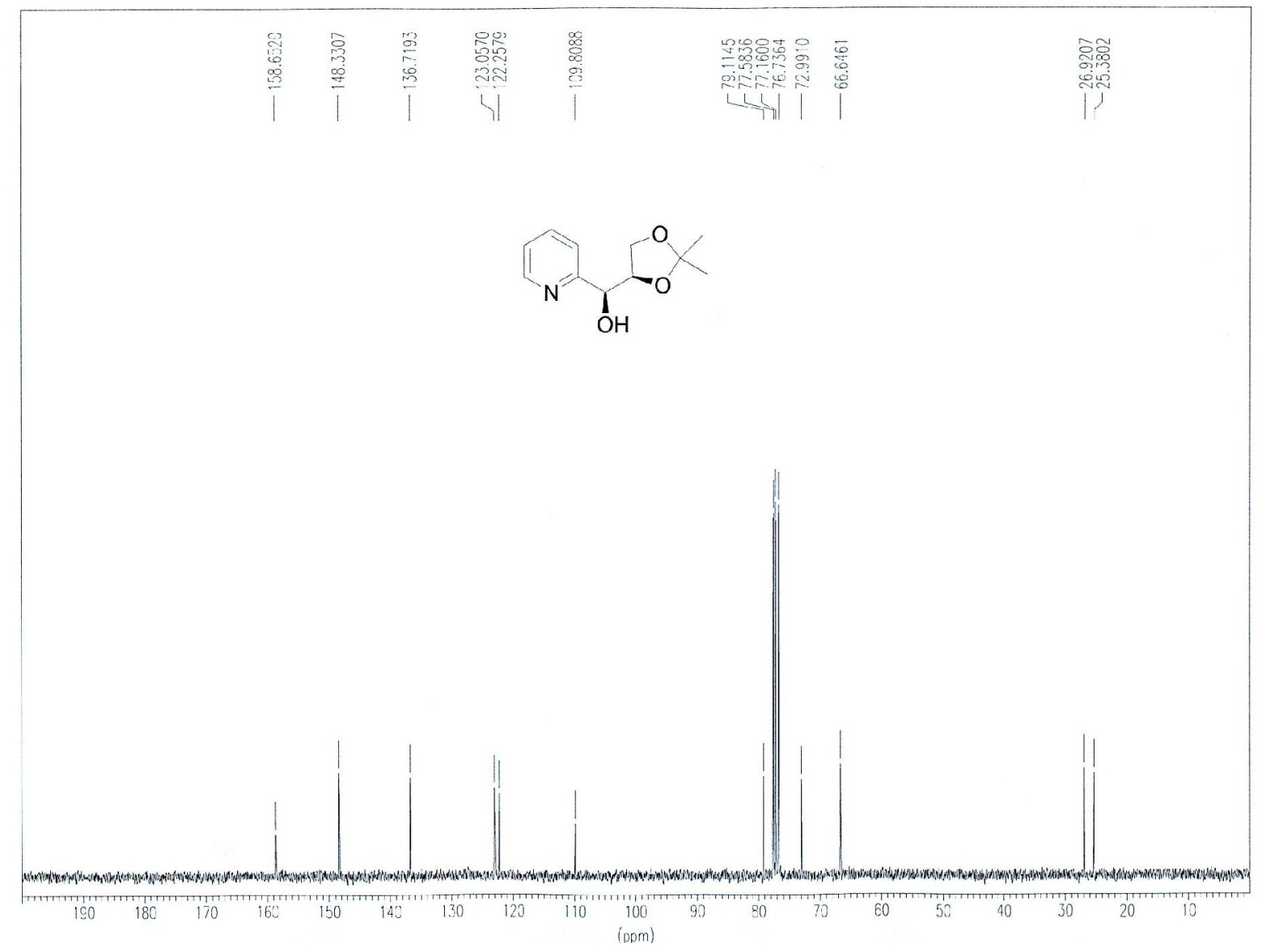

(1R,2R)-2,3-O-Isopropylidene-1-(2'-pyridyl)-1,2,3 trihydroxypropane 2

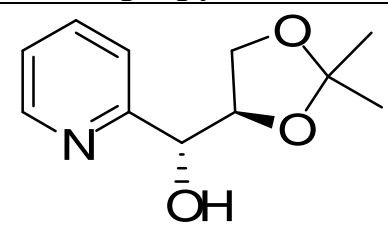

Mol. Formula : $\mathrm{C}_{11} \mathrm{H}_{15} \mathrm{NO}_{3}$

Mol. Weight : 209.24

(Minor diastereoisomer from previous experiment)

${ }^{1} \mathrm{H}$ NMR $\left(\mathrm{CDCl}_{3}\right): 1.30$ (s, 3H), $1.36(\mathrm{~s}, 3 \mathrm{H}), 3.88(\mathrm{~m}, 2 \mathrm{H}), 4.10$ (br s, $\left.1 \mathrm{H}\right), 4.39$ (dt, J=6.4, $5.4,1 \mathrm{H}), 4.72(\mathrm{~d}, J=5.4,1 \mathrm{H}), 7.17(\mathrm{dd}, J=7.6,4.8,1 \mathrm{H}), 7.44$ (d, $J=7.6,1 \mathrm{H}), 7.70$ (dt, $J=7.6$, $1.5,1 \mathrm{H}), 8.49(\mathrm{~d}, J=4.8,1 \mathrm{H})$

${ }^{13} \mathrm{C} \mathrm{NMR}\left(\mathrm{CDCl}_{3}\right): 25.2,26.4,65.8,73.7,78.9,109.6,121.6,122.9,136.6,148.5,158.7$

$[\alpha]_{D}^{20}=-29\left(c 1, \mathrm{CHCl}_{3}\right)$

$\mathrm{IC}(+) \mathrm{MS}(\mathrm{MH}+) \mathrm{m} / \mathrm{z}=210(100 \%), 154,117,73$

Anal. calcd for $\mathrm{C}_{11} \mathrm{H}_{15} \mathrm{NO}_{3}: \mathrm{C}, 63.14 ; \mathrm{H}, 7.23 ; \mathrm{N}, 6.69$ found $\mathrm{C}, 62.80 ; \mathrm{H}, 7.67 ; \mathrm{N}, 6.18$ 


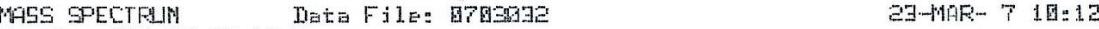

Sarple: PRI GE GI tEuH

-
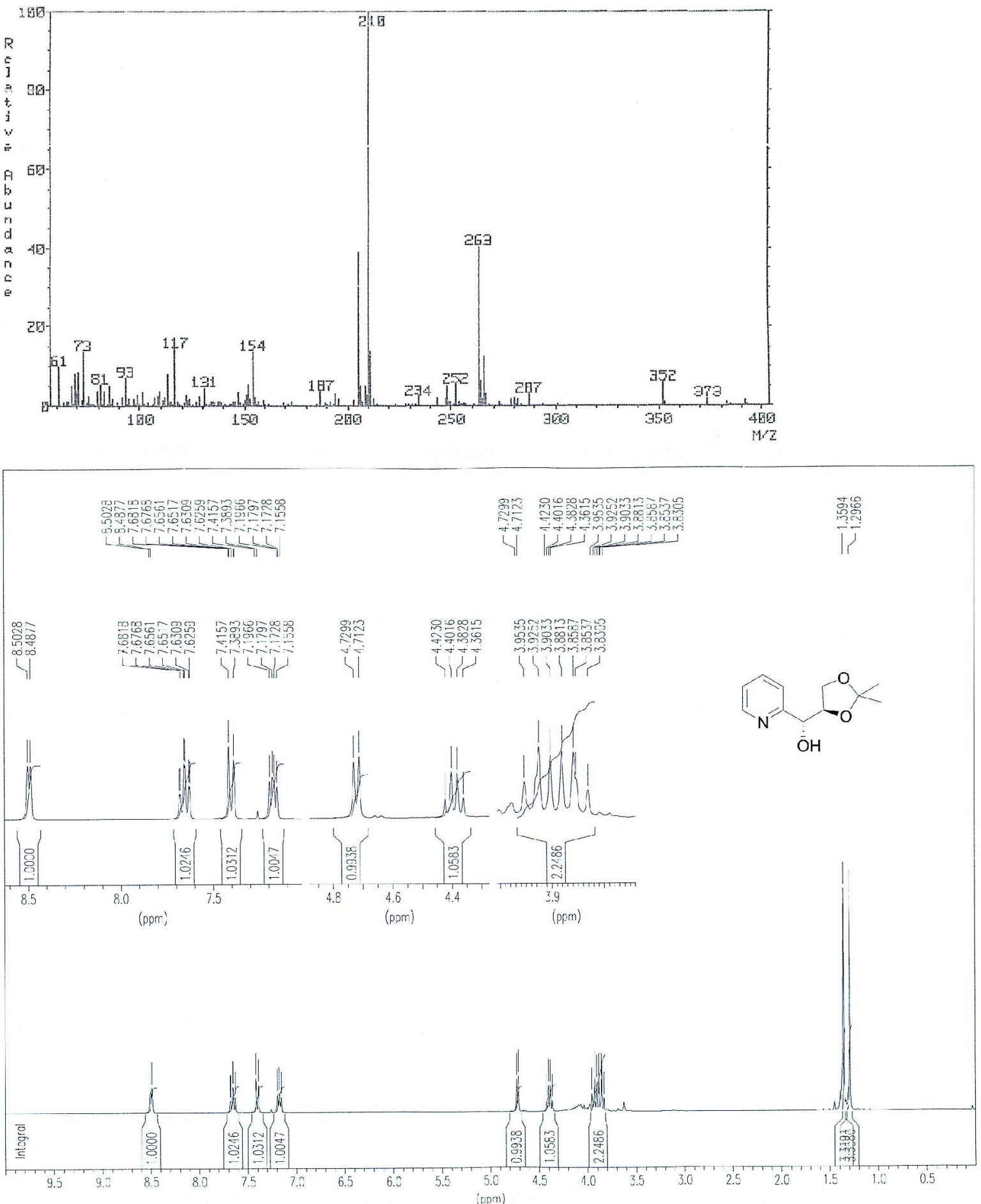


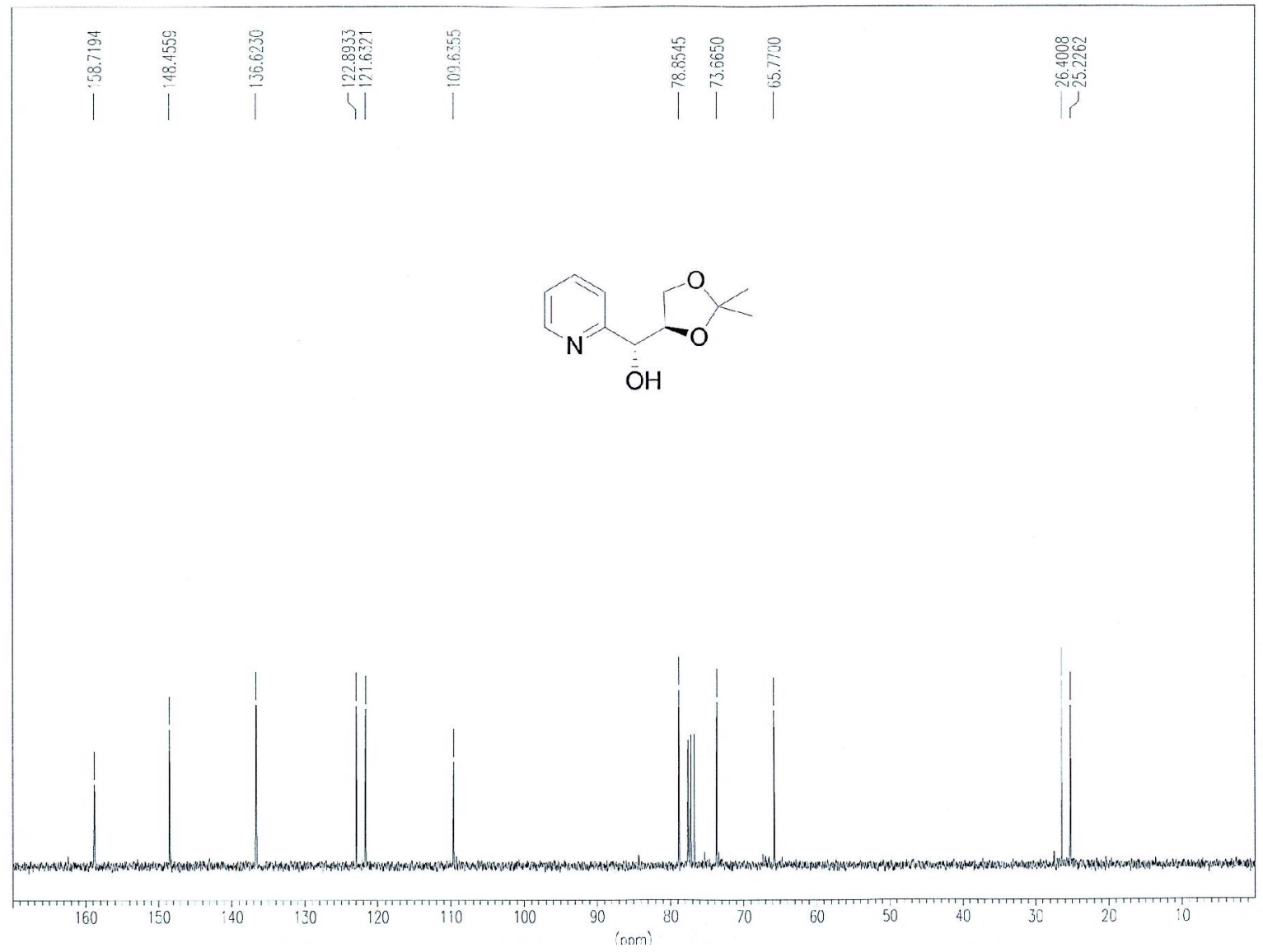

(4R)-2,2-dimethyl-1,3-dioxolane-4-carbaldehyde $\mathbf{3}$ (procedure described in ref 8)

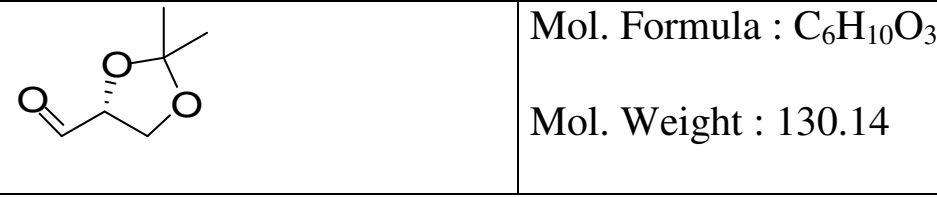

To a solution of 1,2,5,6-diisopropylidene-(D)-mannitol (2 g, $7.62 \mathrm{mmol})$ in dichloromethane $(18 \mathrm{~mL})$ were added saturated $\mathrm{NaHCO}_{3}(0.8 \mathrm{~mL})$, followed by $\mathrm{NaIO}_{4}(3.26 \mathrm{~g}, 15 \mathrm{mmol})$, and the mixture was stirred for $1.5 \mathrm{~h}$ at room temperature. After extractive workup with dichloromethane, solvent was evaporated and the residue was purified by distillation under reduced pressure $\left(26^{\circ} \mathrm{C}, 9 \mathrm{~mm} \mathrm{Hg}\right)$. Aldehyde $3(1.9 \mathrm{~g}, 96 \%)$ was obtained as a colorless oil.

${ }^{1} \mathrm{H} \mathrm{NMR}\left(\mathrm{CDCl}_{3}\right): 1.41(\mathrm{~s}, 3 \mathrm{H}), 1.48(\mathrm{~s}, 3 \mathrm{H}), 4.13(\mathrm{~m}, 2 \mathrm{H}), 4.38(\mathrm{~m}, 1 \mathrm{H}), 9.71(\mathrm{~d}, J=1.7,1 \mathrm{H})$.

${ }^{13} \mathrm{C} \mathrm{NMR}\left(\mathrm{CDCl}_{3}\right): 25.3,26.4,65.7,80,11.4,202$

$[\alpha]_{D}^{20}=+53.8\left(c 2, \mathrm{CHCl}_{3}\right)$

$\mathrm{IC}(+) \mathrm{MS}(\mathrm{MH}+) \mathrm{m} / \mathrm{z}=131,115,101(100 \%), 85$

HRMS calcd for $\mathrm{C}_{6} \mathrm{H}_{11} \mathrm{O}_{3}(\mathrm{MH}+)$ : 131.0708 found 131.0713 

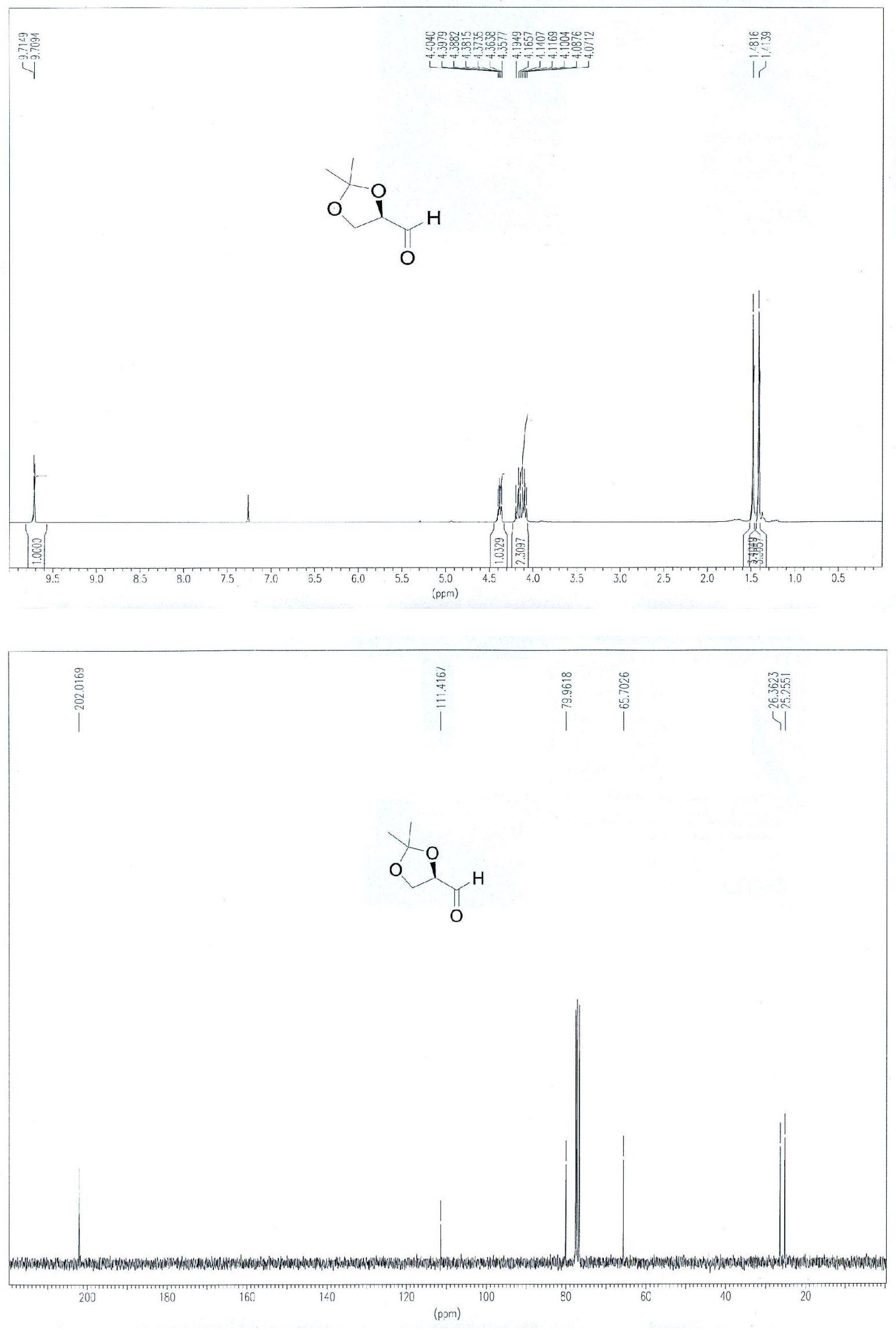
<smiles>OC[C@H](O)[C@H](O)c1ccc[nH]1</smiles>

Mol. Formula : $\mathrm{C}_{8} \mathrm{H}_{12} \mathrm{ClNO}_{3}$

Mol. Weight : 205.64

Compound 1 (450 mg, $2.15 \mathrm{mmol})$ was taken up in dioxane $(4 \mathrm{~mL})$ and an equal volume of $2 \mathrm{M}$ aq. $\mathrm{HCl}$ was added. After $2 \mathrm{~h}$ stirring at room temperature, solvents were evaporated in vacuo and the residue was dissolved in $\mathrm{H}_{2} \mathrm{O}$, washed with diethylether and freeze-dried to afford pure (1S,2R)-1-(2'-pyridyl)-1,2,3 trihydroxypropane 4 (420 $\mathrm{mg}, 95 \%)$ as its hydrochloride salt.

${ }^{1} \mathrm{H}$ NMR $\left(\mathrm{D}_{2} \mathrm{O}\right): 3.67(\mathrm{~m}, 2 \mathrm{H}), 3.93(\mathrm{dt}, J=6.0,4.7,1 \mathrm{H}), 5.1(\mathrm{~d}, J=6.0,1 \mathrm{H}), 7.95(\mathrm{t}, J=7.6$, $1 \mathrm{H}), 8.03(\mathrm{~d}, J=7.6,1 \mathrm{H}), 8.54(\mathrm{t}, J=7.6,1 \mathrm{H}), 8.69(\mathrm{~d}, J=5.8,1 \mathrm{H})$

${ }^{13} \mathrm{C} \mathrm{NMR}\left(\mathrm{CDCl}_{3}\right): 61.9,70.5,73.9,126.1,126.5,140.9,147,155.3$

$[\alpha]_{D}^{20}=-4\left(c 1, \mathrm{CH}_{3} \mathrm{OH}\right)$

$\mathrm{IC}(+) \mathrm{MS}(\mathrm{MH}+) \mathrm{m} / \mathrm{z}=170(100 \%), 154,117,108,79$

Anal. calcd for $\mathrm{C}_{11} \mathrm{H}_{15} \mathrm{NO}_{3}$ : C, 46.73; H, 5.88; N, 6.81 found $\mathrm{C}, 45.94 ; \mathrm{H}, 5.79 ; \mathrm{N}, 6.20$

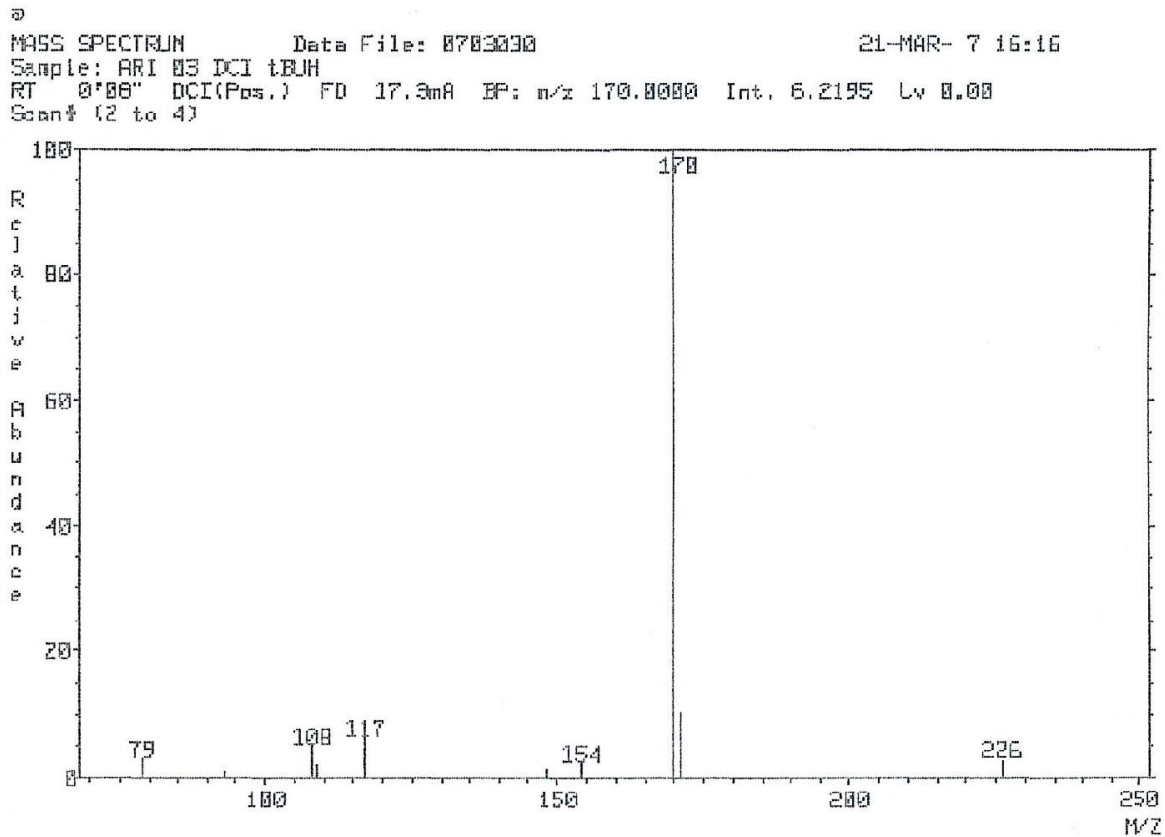



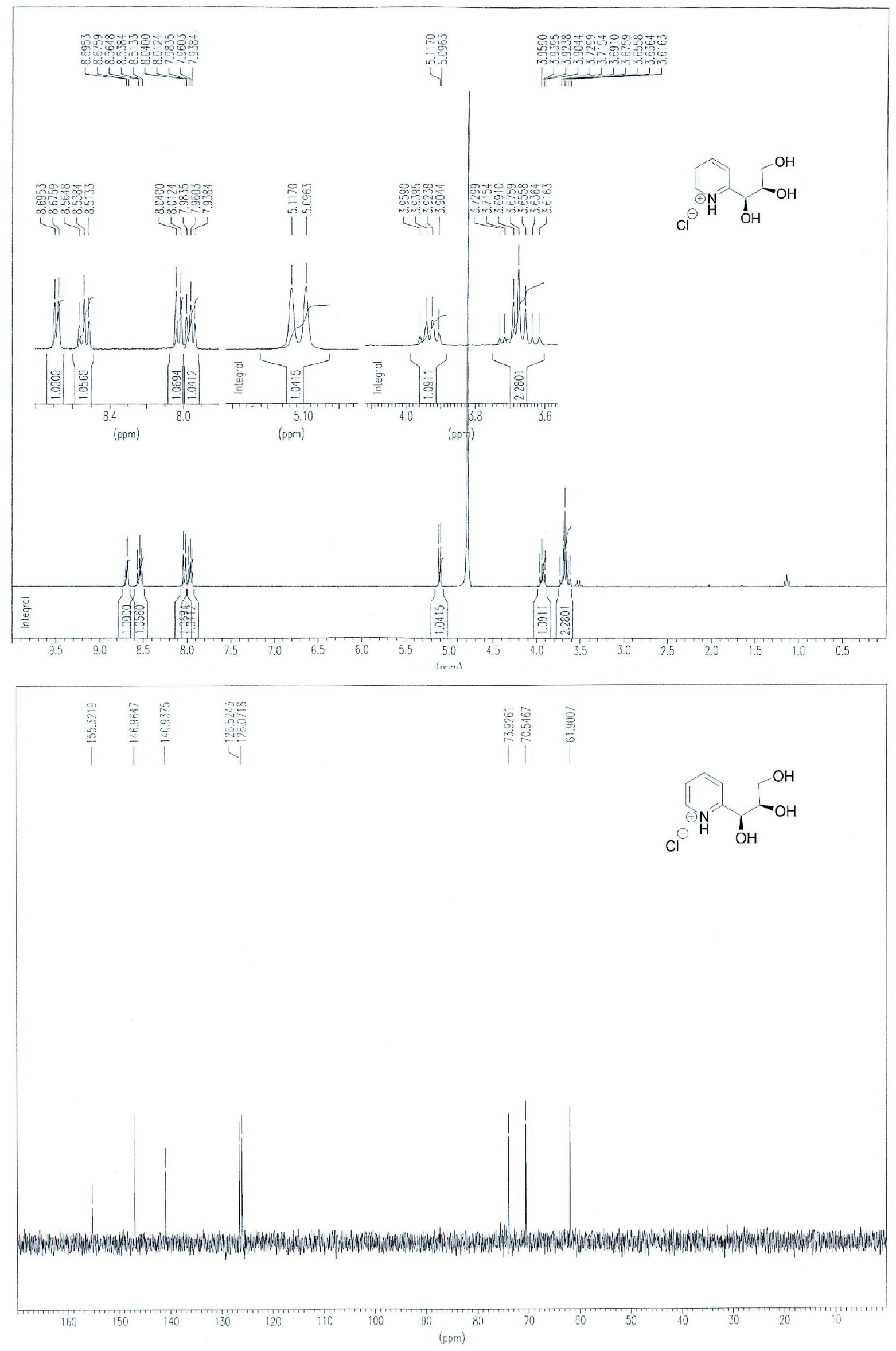


\begin{tabular}{l|l} 
Mol. Formula : $\mathrm{C}_{8} \mathrm{H}_{10} \mathrm{ClNO}_{2}$ \\
Mol. Weight : 187.62
\end{tabular}

To a solution of $4(350 \mathrm{mg}, 1.7 \mathrm{mmol})$ and triphenylphosphine $(669 \mathrm{mg}, 2.55 \mathrm{mmol})$ in anhydrous $\mathrm{CH}_{3} \mathrm{CN}(30 \mathrm{~mL})$ was added diisopropylazodicarboxylate (DIAD) $(0.51 \mathrm{~mL}, 2.55$ mmol) dropwise at $0^{\circ} \mathrm{C}$. after stirring $2 \mathrm{~h}$ at room temperature, $\mathrm{CH}_{3} \mathrm{CN}$ was evaporated and the residue was dissolved in $\mathrm{H}_{2} \mathrm{O}$, washed with $\mathrm{Et}_{2} \mathrm{O}$ and freeze-dried to afford pure 5 (293 mg, $92 \%)$.

${ }^{1} \mathrm{H}$ NMR $\left(\mathrm{D}_{2} \mathrm{O}\right): 4.8(\mathrm{~m}, 1 \mathrm{H}), 4.87(\mathrm{~m}, 2 \mathrm{H}), 5.53(\mathrm{~d}, J=4.5,1 \mathrm{H}), 7.97(\mathrm{t}, J=8,1 \mathrm{H}), 8.11(\mathrm{~d}$, $J=8,1 \mathrm{H}), 8.55(\mathrm{t}, J=8,1 \mathrm{H}), 8.8(\mathrm{~d}, J=6.2,1 \mathrm{H})$.

${ }^{13} \mathrm{C} \mathrm{NMR}\left(\mathrm{CDCl}_{3}\right): 63.5,70.7,74.5,124.9,127.5,141.8,146.7,157.1$.

$\mathrm{IC}(+) \mathrm{MS}(\mathrm{MH}+) \mathrm{m} / \mathrm{z}=190,134(100 \%), 108,73$.

Anal. calcd for $\mathrm{C}_{8} \mathrm{H}_{10} \mathrm{ClNO}_{2}$ : C, 51.21; H, 5.37; N, 7.47 found $\mathrm{C}, 50.80 ; \mathrm{H}, 5.65 ; \mathrm{N}, 6.86$.

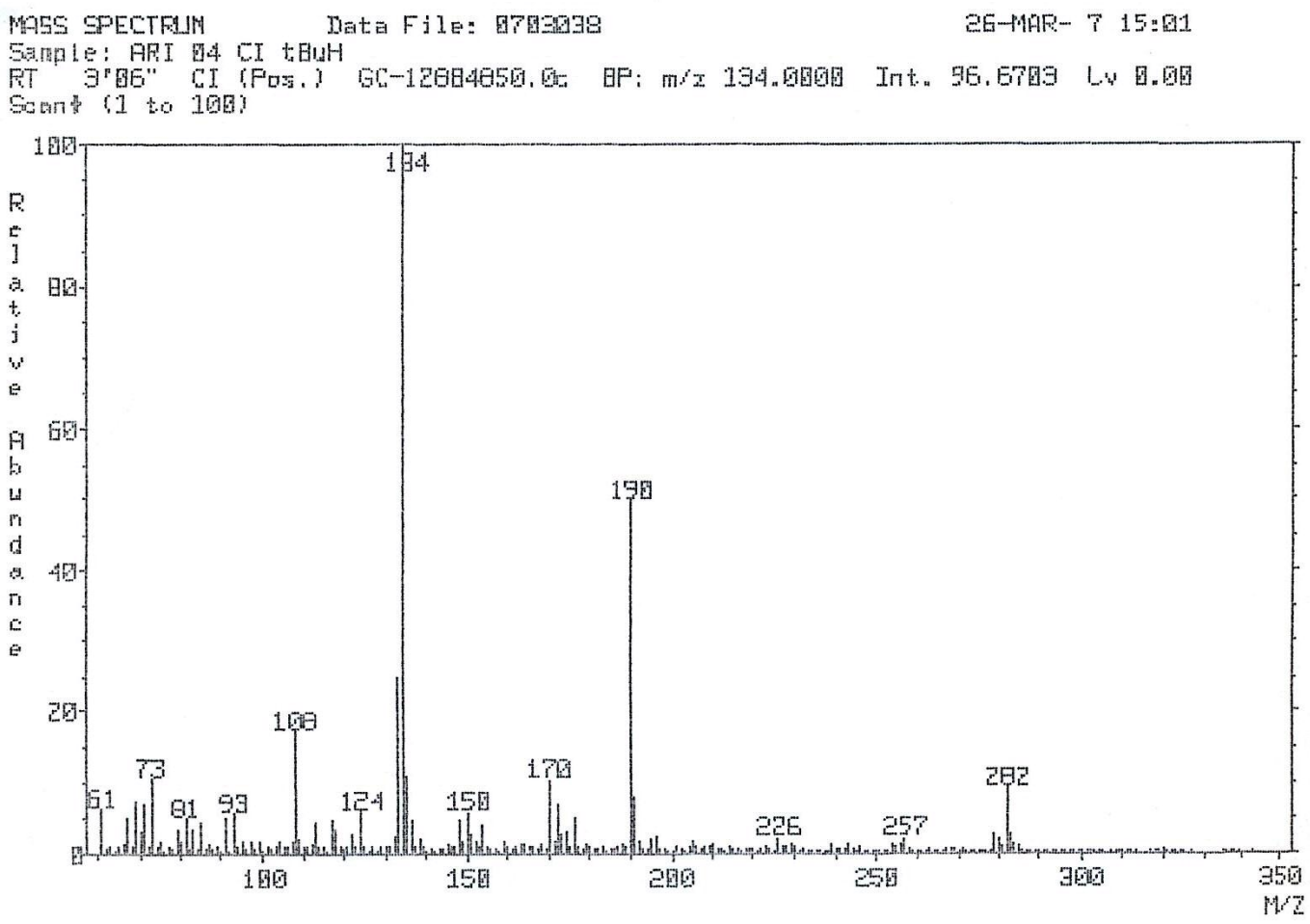



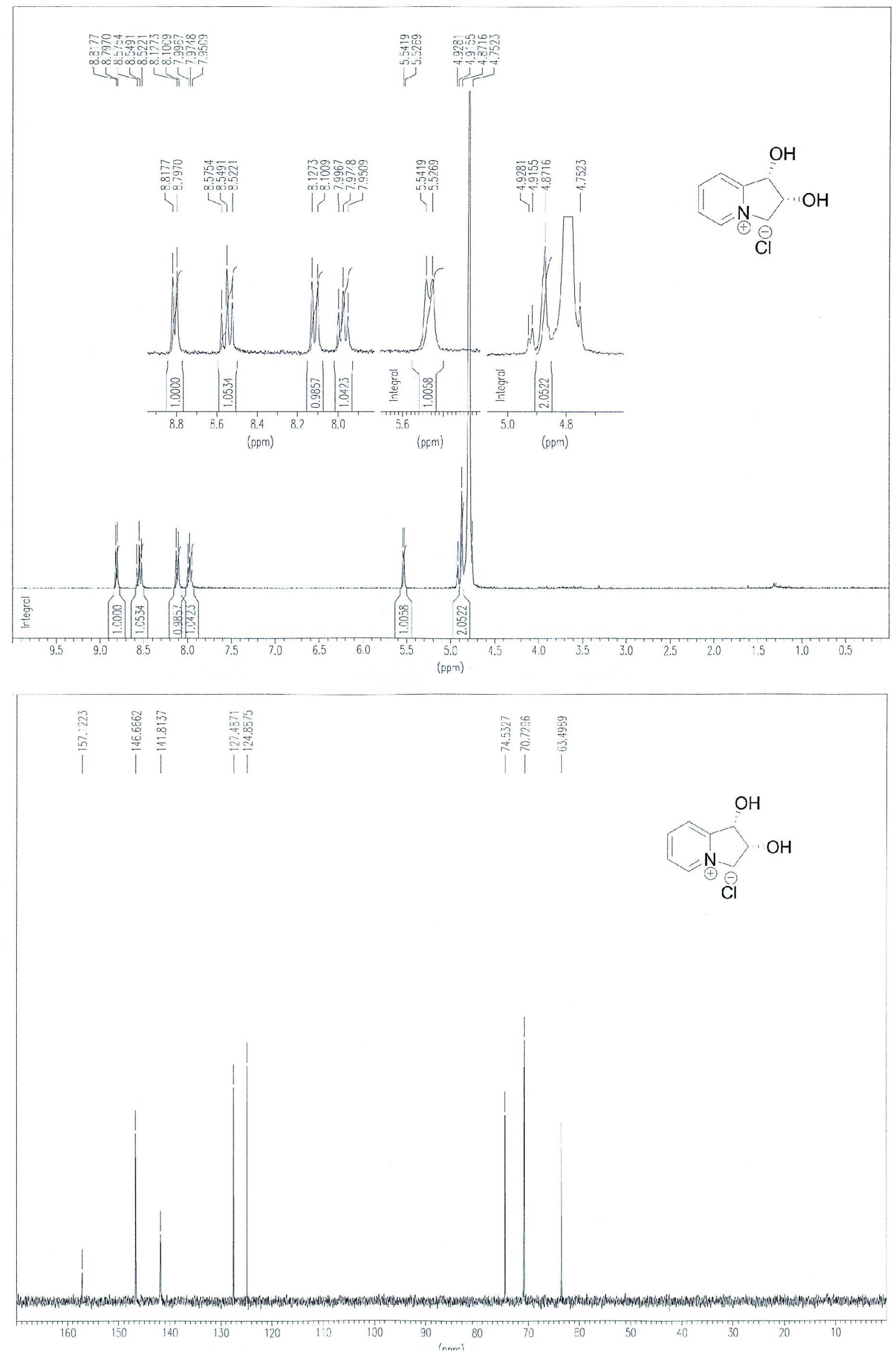
(1S,2R,8aR)-1,2-dihydroxy-octahydro-1H-indolizinium chloride 6

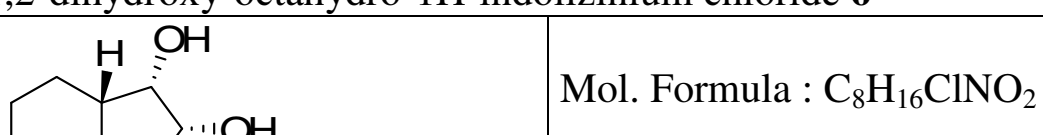

Mol. Weight : 193.67

A solution of (1S,2R)-1,2-dihydroxy-2,3-dihydro-1H-indolizinium chloride 5 (240 mg, 1.28 $\mathrm{mmol})$, in ethanol $(20 \mathrm{~mL})$ was stirred at room temperature in the presence of $\mathrm{PtO}_{2} \cdot \mathrm{H}_{2} \mathrm{O}(41.8$ $\mathrm{mg}, 10 \% \mathrm{~mol}$ ) under an atmospheric pressure of hydrogen. Filtration through a celite pad and evaporation in vacuo afforded compound 6 (231 $\mathrm{mg}, 93 \%$ yield) as a white powder.

${ }^{1} \mathrm{H}$ NMR $\left(\mathrm{D}_{2} \mathrm{O}\right): 1.4-2.1(\mathrm{~m}, 6 \mathrm{H}), 2.9(\mathrm{t}, J=11.8,1 \mathrm{H}), 3.2(\mathrm{~d}, J=11.7,1 \mathrm{H}), 3.6(\mathrm{~m}, 1 \mathrm{H}), 3.4(\mathrm{~m}$, $2,2 \mathrm{H}), 4.3(\mathrm{t}, J=2.1,2 \mathrm{H}), 4.6(\mathrm{~m}, 1 \mathrm{H})$

${ }^{13} \mathrm{C} \mathrm{NMR}\left(\mathrm{CDCl}_{3}\right): 21.6,23,23.1,52.5,57.8,68.2,69.3,70.6$

$[\alpha]_{D}^{20}=-25\left(c 1, \mathrm{CH}_{3} \mathrm{OH}\right)$

$\mathrm{IC}(+) \mathrm{MS}(\mathrm{MH}+) \mathrm{mz} \quad=158(100 \%)$

Anal. calcd for $\mathrm{C}_{8} \mathrm{H}_{16} \mathrm{CNO}{ }_{2}: \mathrm{C}, 49.61 ; \mathrm{H}, 8.33 ; \mathrm{N}, 7.23$ found $\mathrm{C}, 49.12 ; \mathrm{H}, 839 ; \mathrm{N}, 6.96$

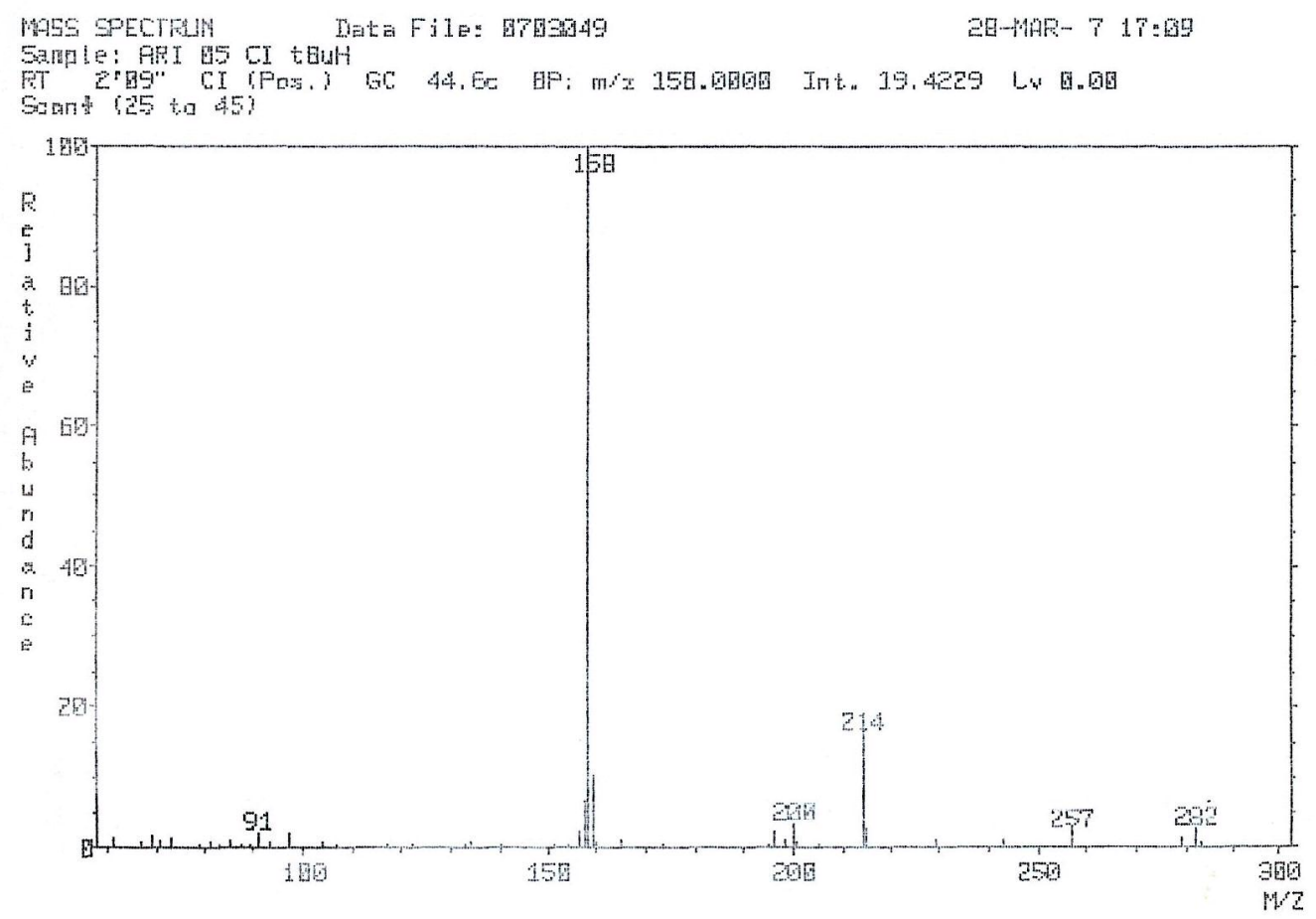



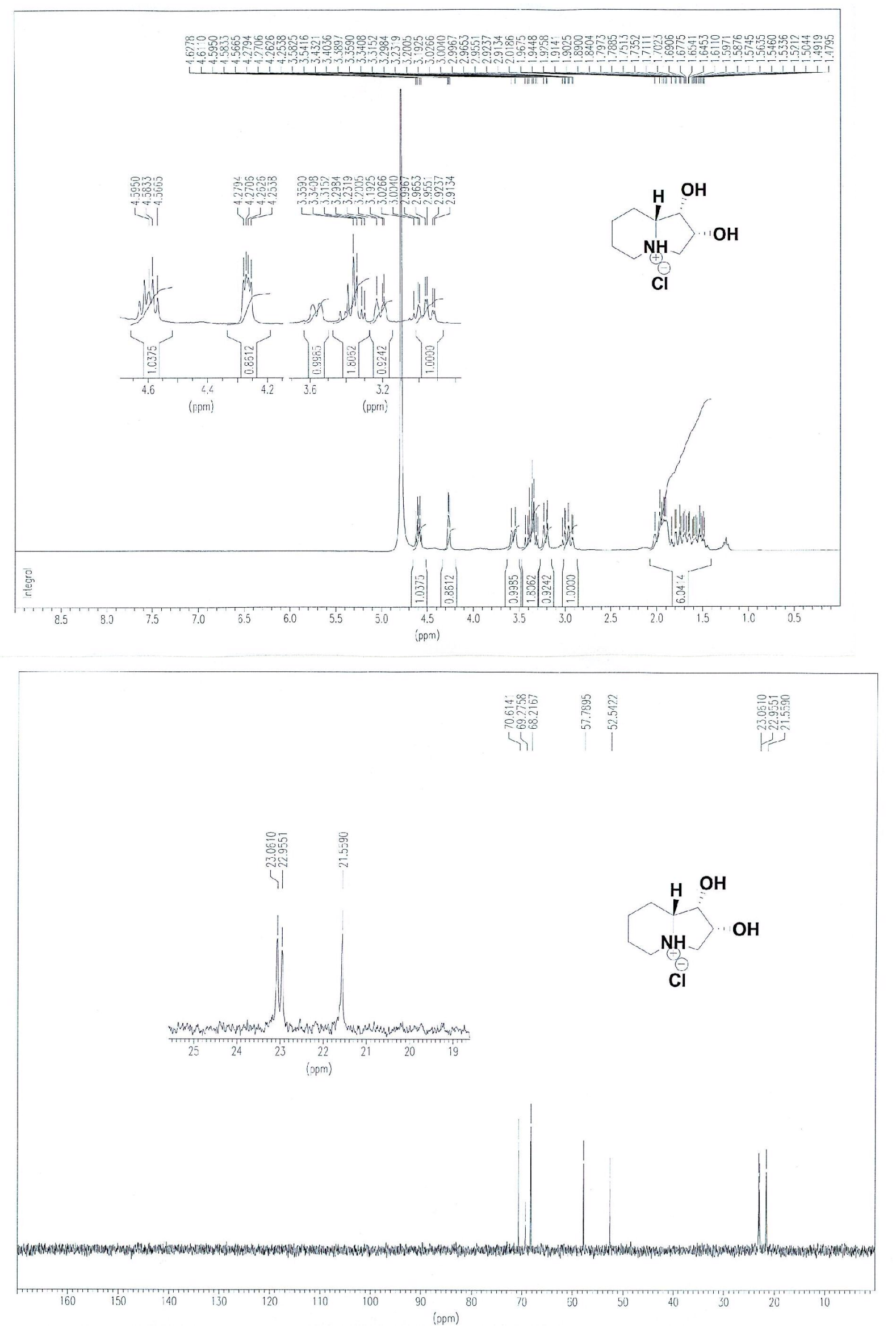


Mol. Formula : $\mathrm{C}_{8} \mathrm{H}_{15} \mathrm{NO}_{2}$

The hydrochloride $6(180 \mathrm{mg}, 0.93 \mathrm{mmol})$ was dissolved in saturated $\mathrm{KOH}(10 \mathrm{~mL})$ and extracted with THF (3 x $15 \mathrm{~mL}$ ). Drying over $\mathrm{K}_{2} \mathrm{CO}_{3}$ followed by solvent evaporation afforded compound 7 (139 mg, $95 \%)$

${ }^{1} \mathrm{H}$ NMR $\left(\mathrm{CDCl}_{3}\right): 1.20(\mathrm{~m}, 1 \mathrm{H}), 1.51-1.88(\mathrm{~m}, 7 \mathrm{H}), 2.26(\mathrm{~m}, 1 \mathrm{H}), 2.91(\mathrm{~d}, J=10.7,1 \mathrm{H}), 3.01$ $(\mathrm{d}, J=10.7,1 \mathrm{H}), 3.60-3.90($ br s, $2 \mathrm{H}), 3.96(\mathrm{~s}, 1 \mathrm{H}), 4.19(\mathrm{~s}, 1 \mathrm{H})$.

${ }^{13} \mathrm{C} \mathrm{NMR}\left(\mathrm{CDCl}_{3}\right): 23.9,24.9,25.0,53.5,62.8,68.4,69.4,72.3$

$[\alpha]_{D}^{20}=-36\left(c 1, \mathrm{C} \mathrm{H}_{3} \mathrm{OH}\right)\left(\right.$ litt: $\left.[\alpha]_{\underline{\mathrm{D}}}=-37.1, \quad c 055, \quad \underline{\mathrm{CH}}_{3} \underline{\mathrm{OH}}\right) \cdot{ }^{20 \mathrm{a}}$

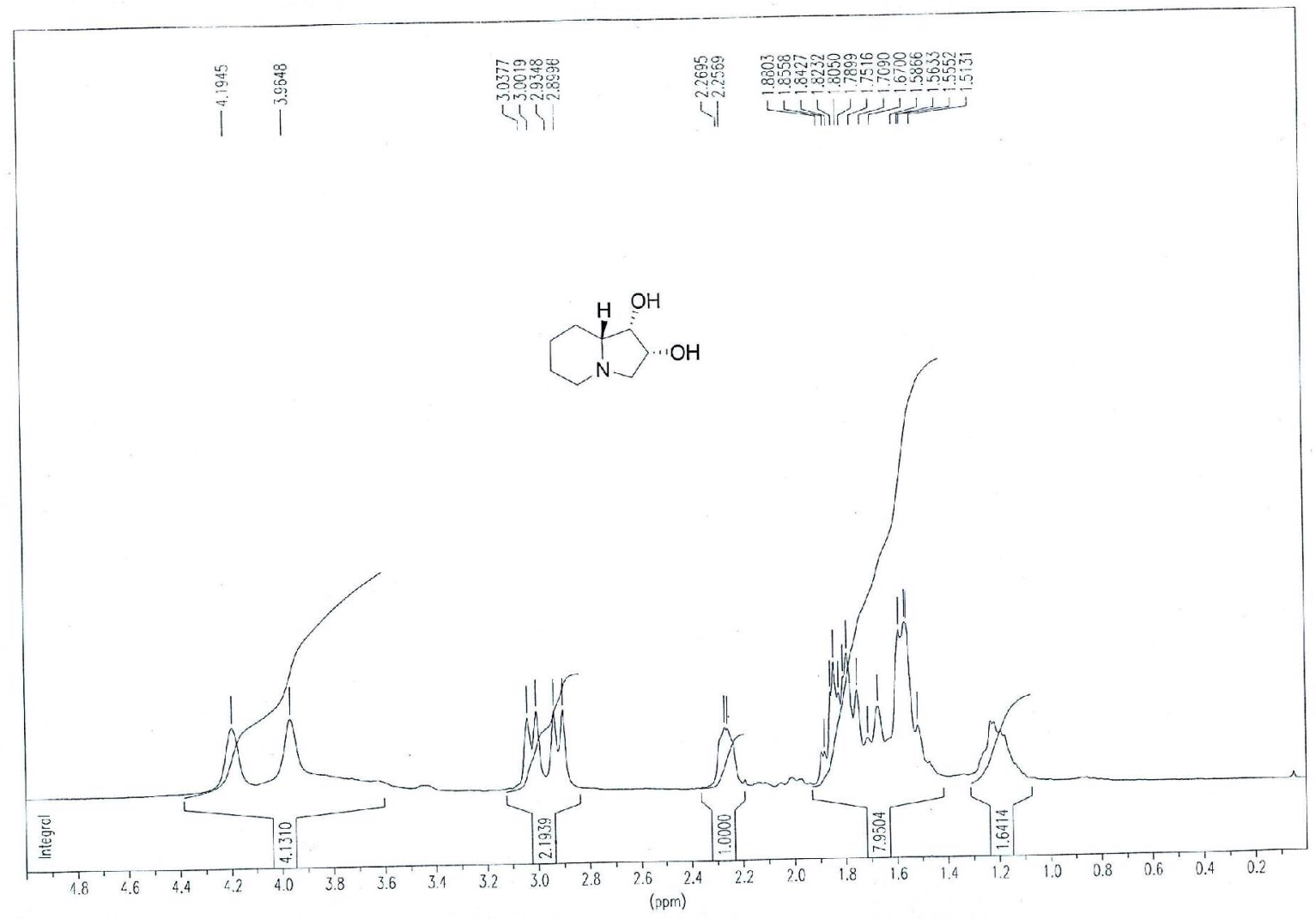




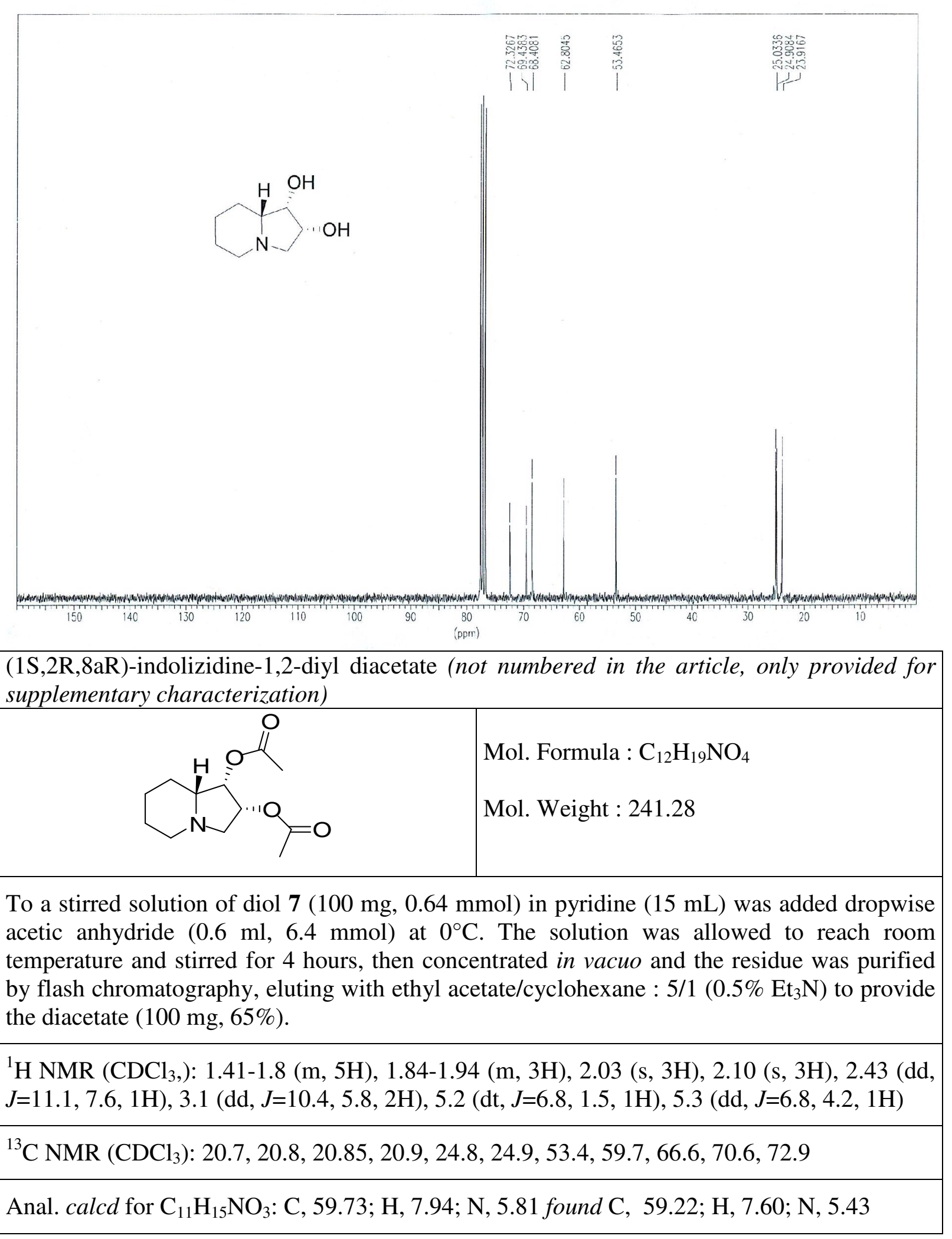



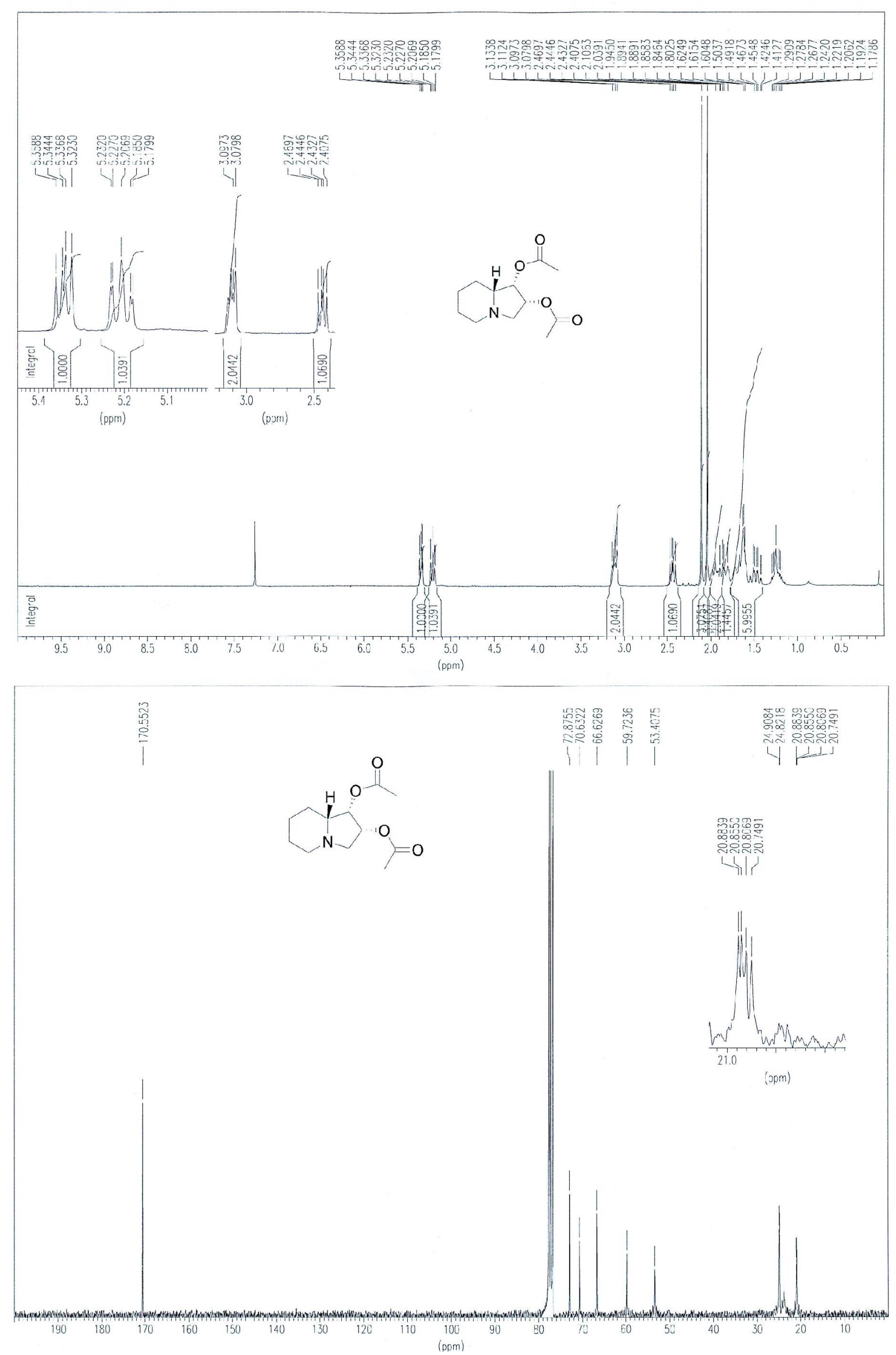
<smiles></smiles>

Mol. Formula : $\mathrm{C}_{8} \mathrm{H}_{12} \mathrm{ClNO}_{3}$

Mol. Weight : 205.64

Applying the procedure described for compound 4, acetonide 2 (200 mg, $0.96 \mathrm{mmol})$ afforded pure triol 8 as a white powder. $\mathrm{m}=195 \mathrm{mg}, 99 \%$

${ }^{1} \mathrm{H}$ NMR $\left(\mathrm{D}_{2} \mathrm{O}\right): 3.66(\mathrm{~m}, 1 \mathrm{H}), 3.79(\mathrm{dd}, J=11.5,5.5,1 \mathrm{H}), 4.0(\mathrm{dt}, J=5.6,2.5,1 \mathrm{H}), 5.28(\mathrm{~d}$, $J=2.25,1 \mathrm{H}), 7.96(\mathrm{t}, J=6.9,1 \mathrm{H}), 8.04(\mathrm{~d}, J=8.28,1 \mathrm{H}), 8.54(\mathrm{dt}, J=7.9,1.3,1 \mathrm{H}), 8.68(\mathrm{~d}$, $J=5.6,1 \mathrm{H})$

${ }^{13} \mathrm{C} \mathrm{NMR}\left(\mathrm{CDCl}_{3}\right): 62.2,69.9,74.2,125.2,126.3,140.7,147.1,156.2$

$[\alpha]_{D}^{20}=-19\left(c 1, \mathrm{CH}_{3} \mathrm{OH}\right)$

$\mathrm{IC}(+) \mathrm{MS}(\mathrm{MH}+) \mathrm{m} / \mathrm{z}=226,212,170(100 \%)$.

Anal. calcd for $\mathrm{C}_{11} \mathrm{H}_{15} \mathrm{NO}_{3}$ : C, 46.73; H, 5.88; N, 6.81 found $\mathrm{C}, 46.44 ; \mathrm{H}, 5.92 ; \mathrm{N}, 6.38$

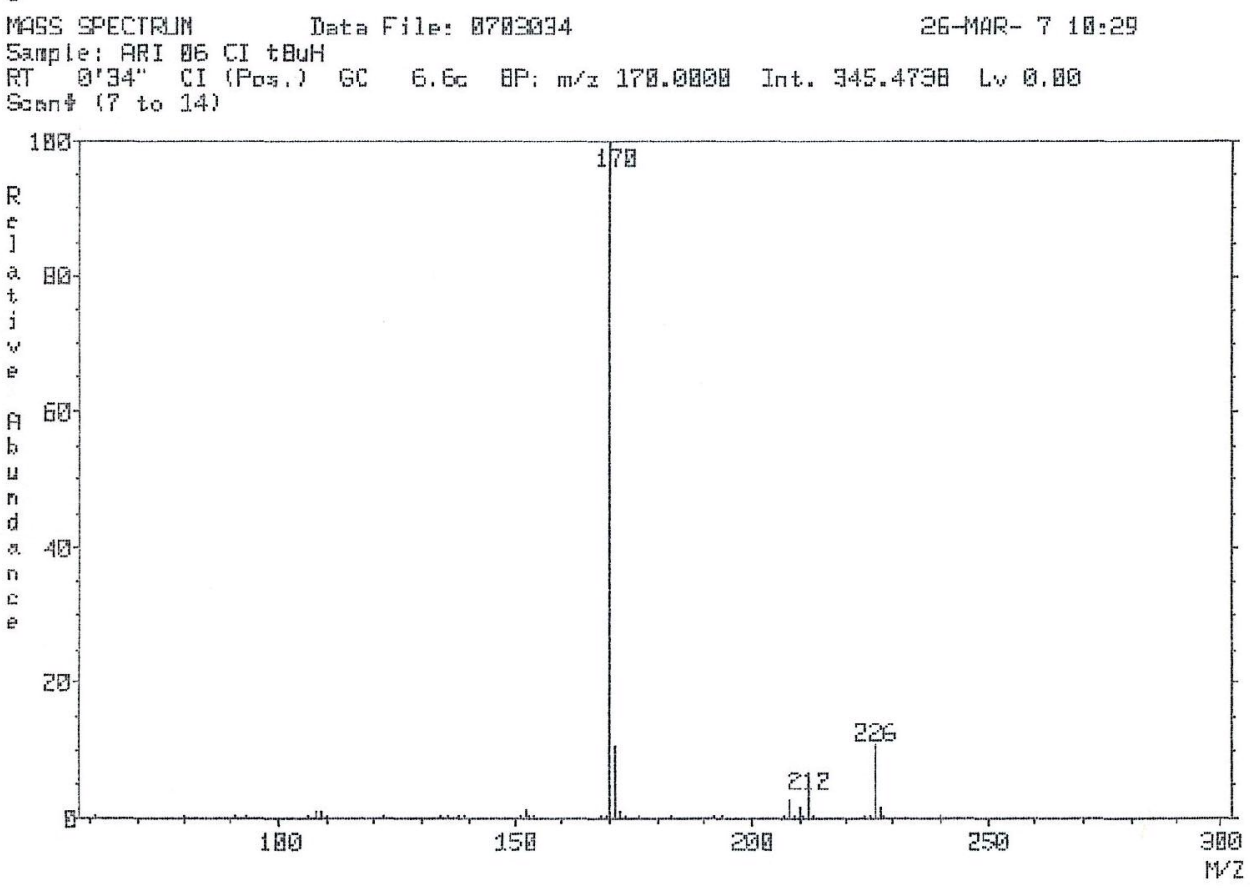



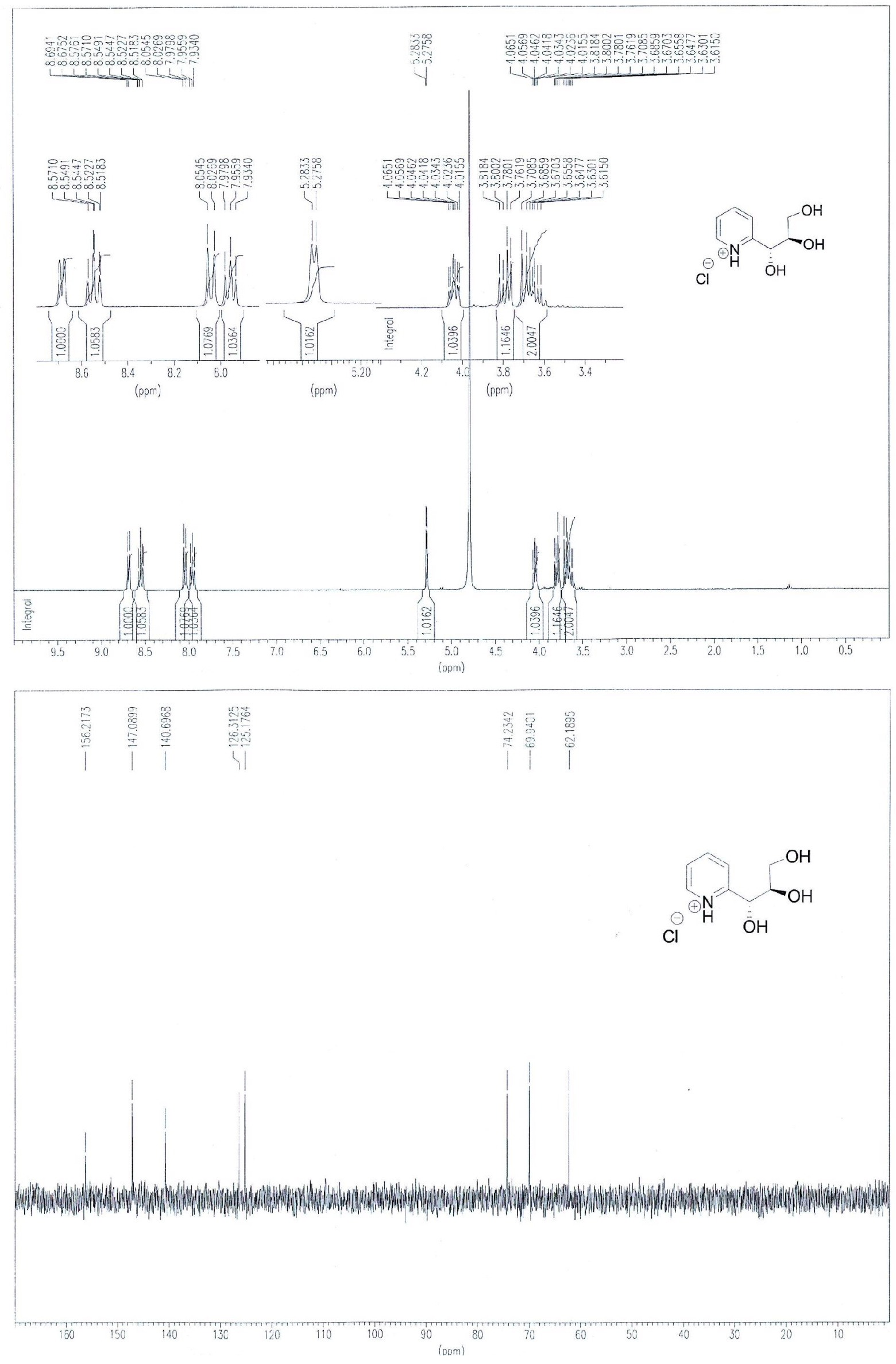
(1R,2R)-1,2-dihydroxy-2,3-dihydro-1H-indolizinium chloride 9.<smiles>OC1C(O)C2CCCCC2(O)C1O</smiles>

Mol. Formula : $\mathrm{C}_{8} \mathrm{H}_{10} \mathrm{ClNO}_{2}$

Mol. Weight : 187.62

Starting from triol 8, the protocol described for compound 5 (160 $\mathrm{mg}, 0.78 \mathrm{mmol})$ gave pure pyridinium 9 (143 $\mathrm{mg}, 98 \%)$ as a white powder.

${ }^{1} \mathrm{H}$ NMR $\left(\mathrm{CDCl}_{3},\right): 4.62(\mathrm{dd}, J=12.9,5.9,1 \mathrm{H}), 4.73(\mathrm{dd}, J=11.9,5.6,1 \mathrm{H}), 5.08(\mathrm{dd}, J=13$, $6.3,1 \mathrm{H}), 5.38(\mathrm{~d}, J=5.5,1 \mathrm{H}), 8.0(\mathrm{t}, J=6.7,1 \mathrm{H}), 8.12(\mathrm{~d}, J=7.9,1 \mathrm{H}), 8.55(\mathrm{t}, J=7.8,1 \mathrm{H}), 8.79$ $(\mathrm{d}, J=6.2,1 \mathrm{H})$.

${ }^{13} \mathrm{C} \mathrm{NMR}\left(\mathrm{CDCl}_{3}\right): 61.4,74.6,77.9,125.2,128,141.7,147,156.5$.

$[\alpha]_{D}^{20}=-19\left(c 1, \mathrm{CH} \quad{ }_{3} \mathrm{OH}\right)$

$\mathbb{E}(70 \mathrm{eV}),(\mathrm{M}+) \mathrm{mz} \quad=152(100 \%) \quad, 131,122,114,99$

Anal. calcd for $\mathrm{C}_{11} \mathrm{H}_{15} \mathrm{NO}_{3}: \mathrm{C}, 5121 ; \mathrm{H}, 537 ; \mathrm{N}, 7.47 \quad$ found $\mathrm{C}, 50.8 \%$ H,52 5; N, 7.06.

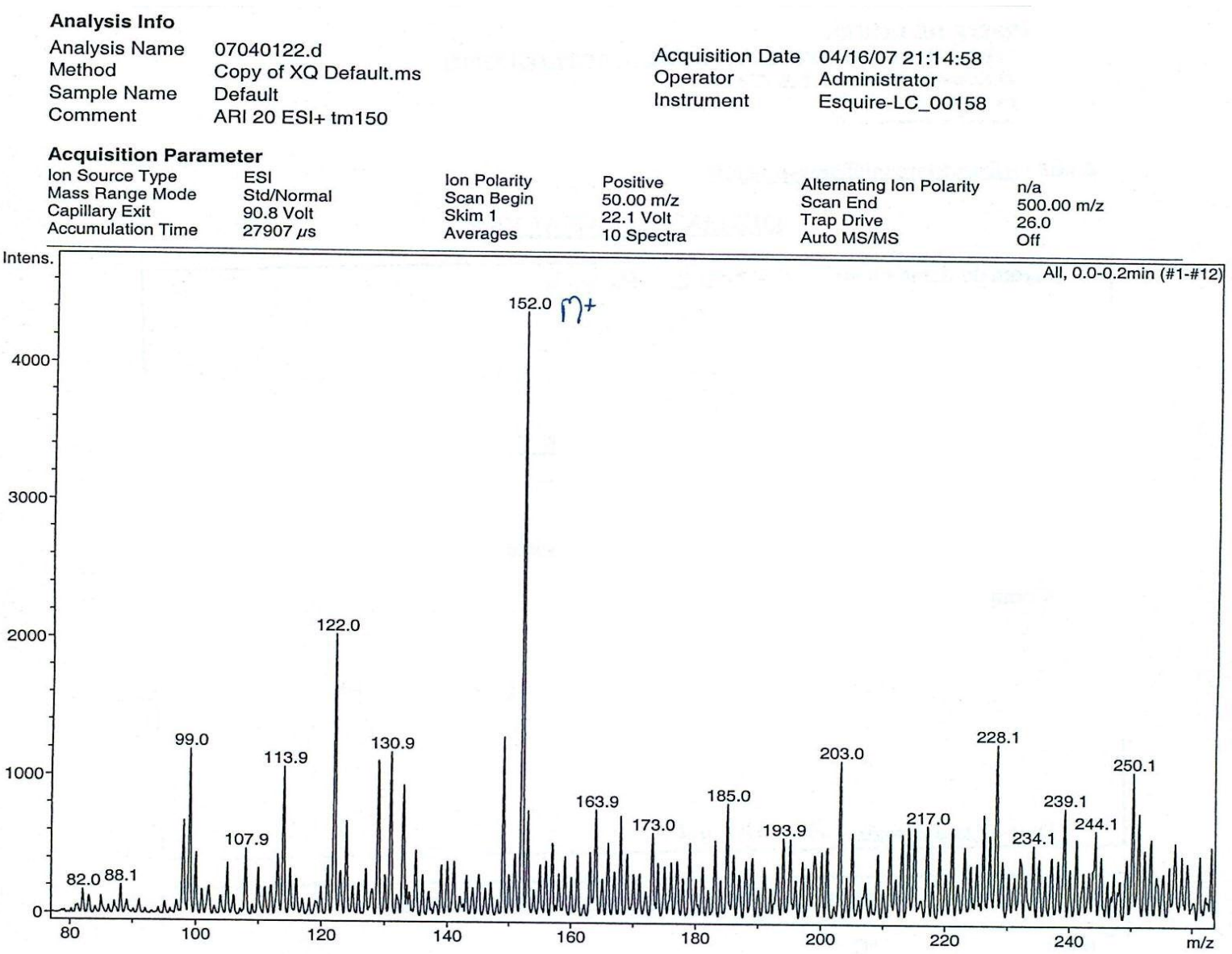



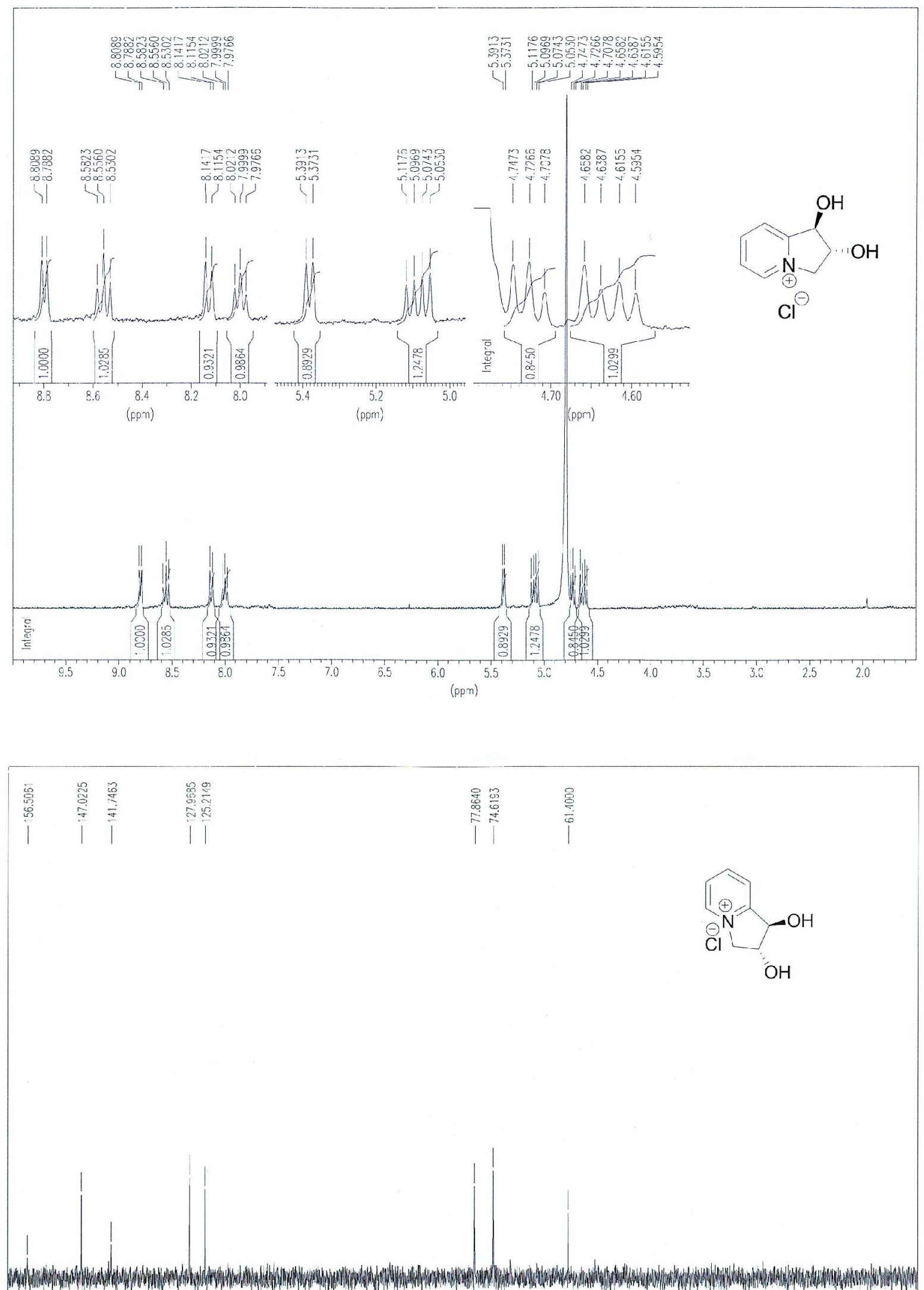

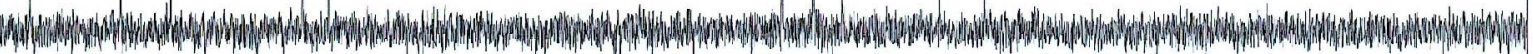

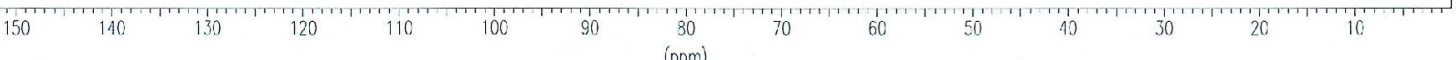


(1R,2R,8aR)-octahydroindolizine-1,2-diol hydrochloride 10a and

(1R,2R,8aS)-octahydroindolizine-1,2-diol hydrochloride $\mathbf{1 0 b}$

\begin{tabular}{l|l}
\hline & Mol. Formula : $\mathrm{C}_{8} \mathrm{H}_{16} \mathrm{ClNO}_{2}$ \\
\hline
\end{tabular}

Using the same procedure described for compound $\mathbf{6}$, the pyridinium salt $9(111 \mathrm{mg}, 0.59$ mmol) provided $\mathbf{1 0 a}+\mathbf{1 0 b}(111 \mathrm{mg}, 97 \%)$ as a white powder

(1R,2R,8aR)-octahydroindolizine-1,2-diol.11a-(1R,2R,8aS)-octahydroindolizine-1,2-diol 11b<smiles>OC1[C@@H](O)CN2CCCC[C@H]12</smiles>

Mol. Formula : $\mathrm{C}_{8} \mathrm{H}_{15} \mathrm{NO}_{2}$

Mol. Weight : 157.21

The hydrochloride $10(61 \mathrm{mg}, 0.32 \mathrm{mmol})$ was dissolved in saturated $\mathrm{KOH}(5 \mathrm{~mL})$ and extracted with THF (3 x $10 \mathrm{~mL}$ ). Drying over $\mathrm{K}_{2} \mathrm{CO}_{3}$ followed by solvent evaporation afforded a mixture of diastereomers 11a and $\mathbf{1 1 b}$ in a 0.92:1 ratio $(47 \mathrm{mg}, 95 \%)$.

${ }^{1} \mathrm{H}$ NMR: ${ }^{21}$

$11 \mathrm{a}\left(\mathrm{D}_{2} \mathrm{O}\right)$ : 1.16-1.42 (m, $\left.3 \mathrm{H}\right), 1.58-1.67$ (m, $\left.1 \mathrm{H}\right), 1.76-1.82(\mathrm{~m}, 1 \mathrm{H}), 1.87-1.91(\mathrm{~m}, 2 \mathrm{H})$, $1.95-2.08(\mathrm{~m}, 1 \mathrm{H}), 2.57(\mathrm{dd}, J=11.2,7.6,1 \mathrm{H}), 2.78$ (dd, $J=11.2,1.4,1 \mathrm{H}), 2.91$ (br d, $J=11.1$ $\mathrm{Hz}, 1 \mathrm{H}), 3.61(\mathrm{dd}, J=8.7,4.0,1 \mathrm{H}), 4.01(\mathrm{~m}, 1 \mathrm{H})$

$11 \mathrm{~b}\left(\mathrm{CDl}_{3}\right): 1.25-1.75(\mathrm{~m}, 5 \mathrm{H}), 1.80-1.89(\mathrm{~m}, 1 \mathrm{H}), 2.03-2.14(\mathrm{~m}, 2 \mathrm{H}), 2.25(\mathrm{~m}, 1 \mathrm{H}), 2.90-$

$3.13(\mathrm{~m}, 3 \underline{\mathrm{H}}), 3.54(\mathrm{dd}, J=9.6,7.3,1 \mathrm{H}), 3.83(\mathrm{~d}, J=4.1,1 \underline{\mathrm{H}}), 4.21(\mathrm{~m}, 1 \mathrm{H})$

${ }^{13} \mathrm{C} \mathrm{NMR}\left(\mathrm{CDCl}_{3}\right):{ }^{21}$

11a: $24.0,24.8,28.7,53.5,61.7,70.0,77.1,84.8$

11b: $23.9,24.5,25.0,53.5,61.7,66.7,77.5,80.5$

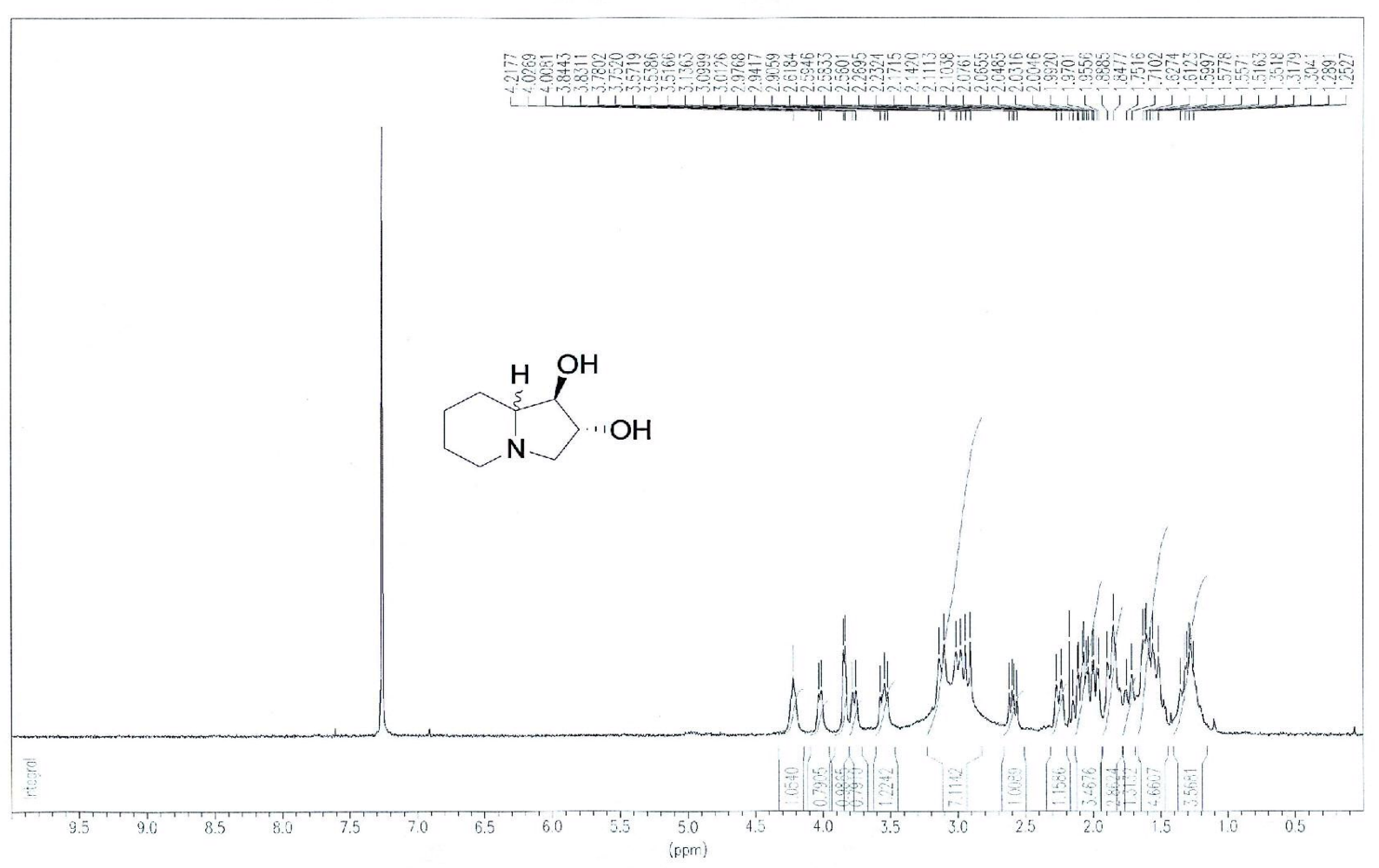



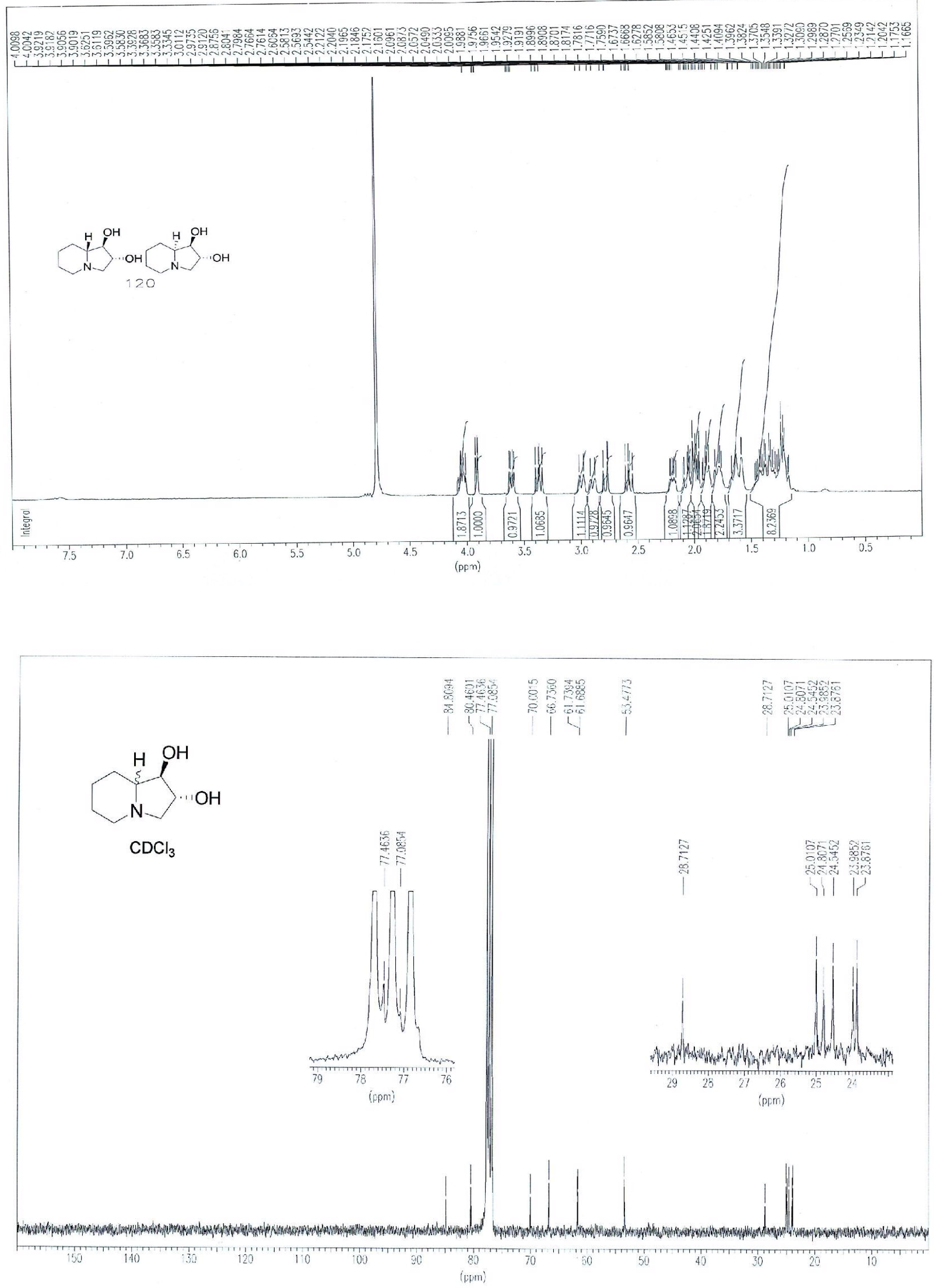
<smiles>Cc1ccc(C(=O)O[C@H](c2ccccn2)C2COC(C)(C)O2)cc1</smiles>

Mol. Formula : $\mathrm{C}_{19} \mathrm{H}_{21} \mathrm{NO}_{4}$

Mol. Weight : 327.37

To a solution of compound $2(113 \mathrm{mg}, 0.54 \mathrm{mmol})$ and $p$-toluoyl chloride $(94 \mu \mathrm{l}, 0.7 \mathrm{mmol})$ in dichloromethane $(15 \mathrm{~mL})$ was added pyridine $(0.13 \mathrm{~mL}, 1.62 \mathrm{mmol})$, at $0^{\circ} \mathrm{C}$ then stirred for 4 hours at room temperature. The solution was evaporated and the residue was purified by flash chromatography. Eluting with EtOAc/cyclohexane $=1 / 2$, provided $\mathbf{1 2}$ as a colorless oil (164 mg, $93 \%)$.

${ }^{1} \mathrm{H}$ NMR $\left(\mathrm{CDCl}_{3}\right): 1.4(\mathrm{~s}, 3 \mathrm{H}), 1.5(\mathrm{~s}, 3 \mathrm{H}), 2.4(\mathrm{~s}, 3 \mathrm{H}), 4(\mathrm{~m}, 2 \mathrm{H}), 4.8(\mathrm{q}, J=6.2,1 \mathrm{H}), 6.1(\mathrm{~d}$, $J=5.6,1 \mathrm{H}), 7.2(\mathrm{dd}, J=5.2,7.6,1 \mathrm{H}), 7.24(\mathrm{~d}, J=8.2,2 \mathrm{H}), 7.4(\mathrm{~d}, J=7.6,1 \mathrm{H}), 7.7(\mathrm{dt}, J=1.5$, $7.7,1 \mathrm{H}), 8.0(\mathrm{~d}, J=8.2,2 \mathrm{H}), 8.6(\mathrm{~d}, J=5.2,1 \mathrm{H})$

${ }^{13} \mathrm{C} \mathrm{NMR}\left(\mathrm{CDCl}_{3}\right): 21.8,25.5,26.6,66,76.9,77,110.1,122.1,123.2,127.1,129.2,130$, $136.8,144.1,149.4,156.9,166$

$\mathrm{IC}(+) \mathrm{MS}\left(\mathrm{MH}^{+}\right) \mathrm{m} / \mathrm{z}=328(100 \%), 270$

HRMS for $\mathrm{C}_{19} \mathrm{H}_{22} \mathrm{NO}_{4}\left(\mathrm{MH}^{+}\right): 328.1549$, found: 328.1567

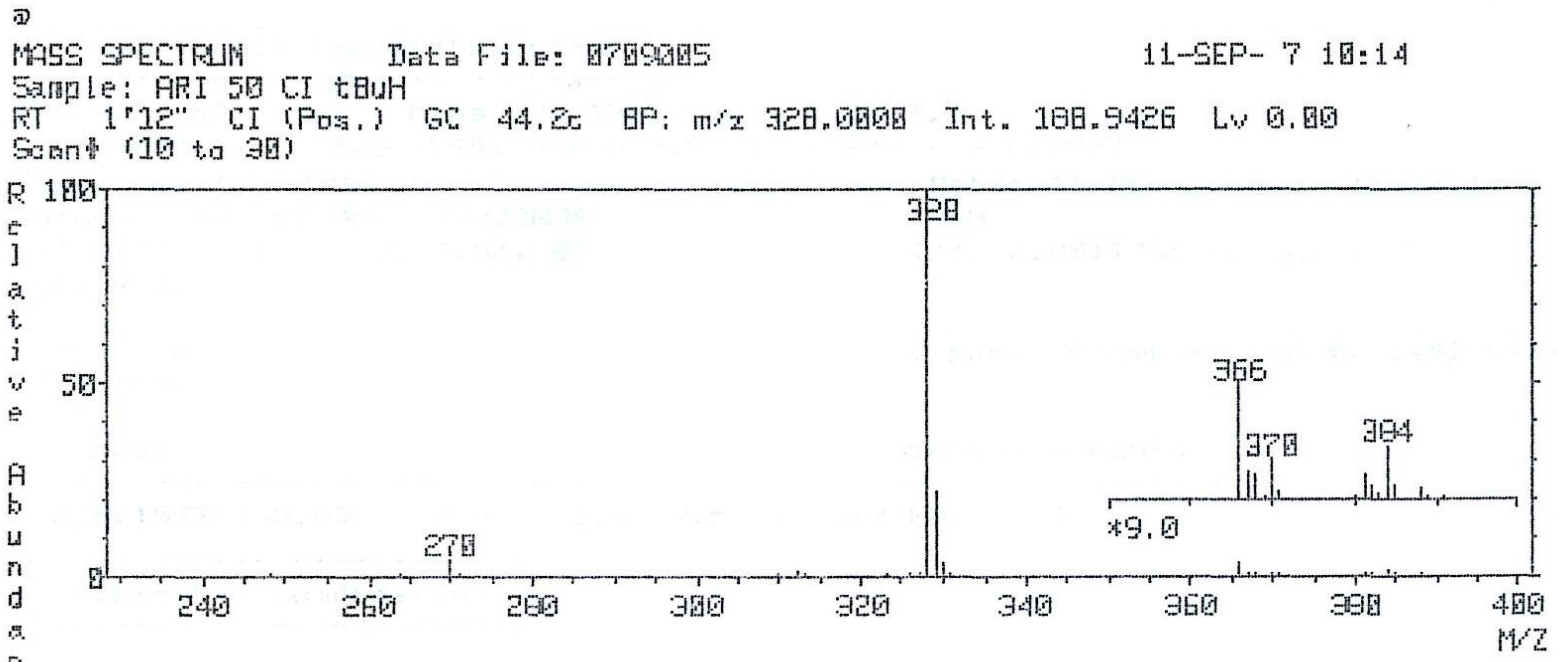




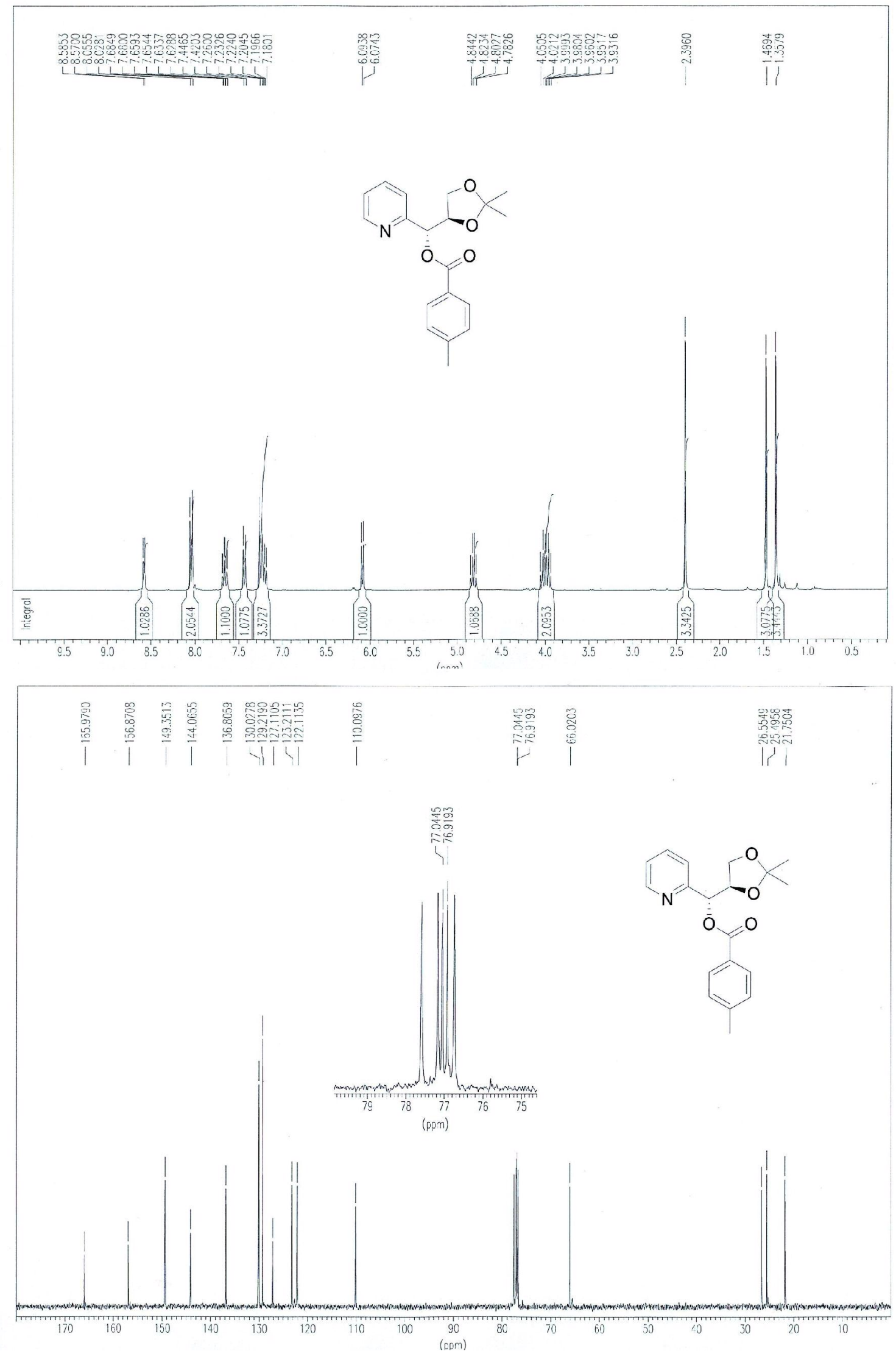




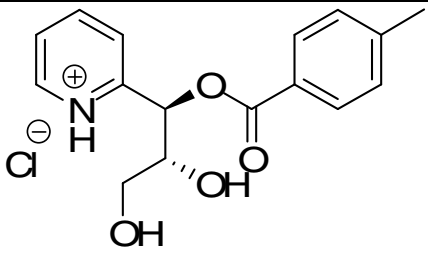

Mol. Formula : $\mathrm{C}_{16} \mathrm{H}_{18} \mathrm{ClNO}_{4}$

Mol. Weight : 323.77

Applying the procedure described for compound 4, acetonide 12 afforded pure diol 13 (139 $\mathrm{mg}, 90 \%)$, as a white powder.

${ }^{1} \mathrm{H}$ NMR $\left(\mathrm{D}_{2} \mathrm{O}\right): 2.4(\mathrm{~s}, 3 \mathrm{H}), 3.8(\mathrm{~m}, 2 \mathrm{H}), 4.2(\mathrm{~d}, J=3.1,1 \mathrm{H}), 6.3(\mathrm{~d}, J=3.1,1 \mathrm{H}), 7.4(\mathrm{~d}, J=8.0$, $2 \mathrm{H}), 7.9(\mathrm{t}, J=6.6,1 \mathrm{H}), 8.0(\mathrm{~d}, J=8.0,2 \mathrm{H}), 8.1(\mathrm{~d}, J=7.9,1 \mathrm{H}), 8.5(\mathrm{t}, \mathrm{J}=7.9,1 \mathrm{H}), 8.7(\mathrm{~d}, \mathrm{~J}=4.9$, $1 \mathrm{H})$

${ }^{1} \mathrm{H}$ NMR $\left(\mathrm{D}_{2} \mathrm{O}\right): 21.2,61.9,72.7,73.6,125.2,125.7,126.8,129.8,130.3,142.7,146.6,146.7$, $152.1,167.5$

$\mathrm{IC}(+) \mathrm{MS}\left(\mathrm{M}^{+}\right.$without chloride ion) $\mathrm{m} / \mathrm{z}=288(100 \%), 282$

Anal. calcd for $\mathrm{C}_{16} \mathrm{H}_{18} \mathrm{ClNO}_{4}$ : C, 59.35; H, 5.60; N, 4.33 found $\mathrm{C}, 59.01 ; \mathrm{H}, 5.65 ; \mathrm{N}, 4.21$

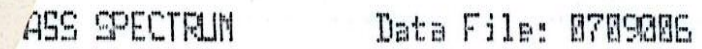

$11-5 E P-718: 32$

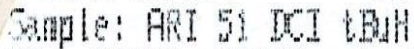

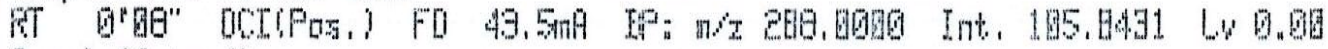

cond 12 to 4

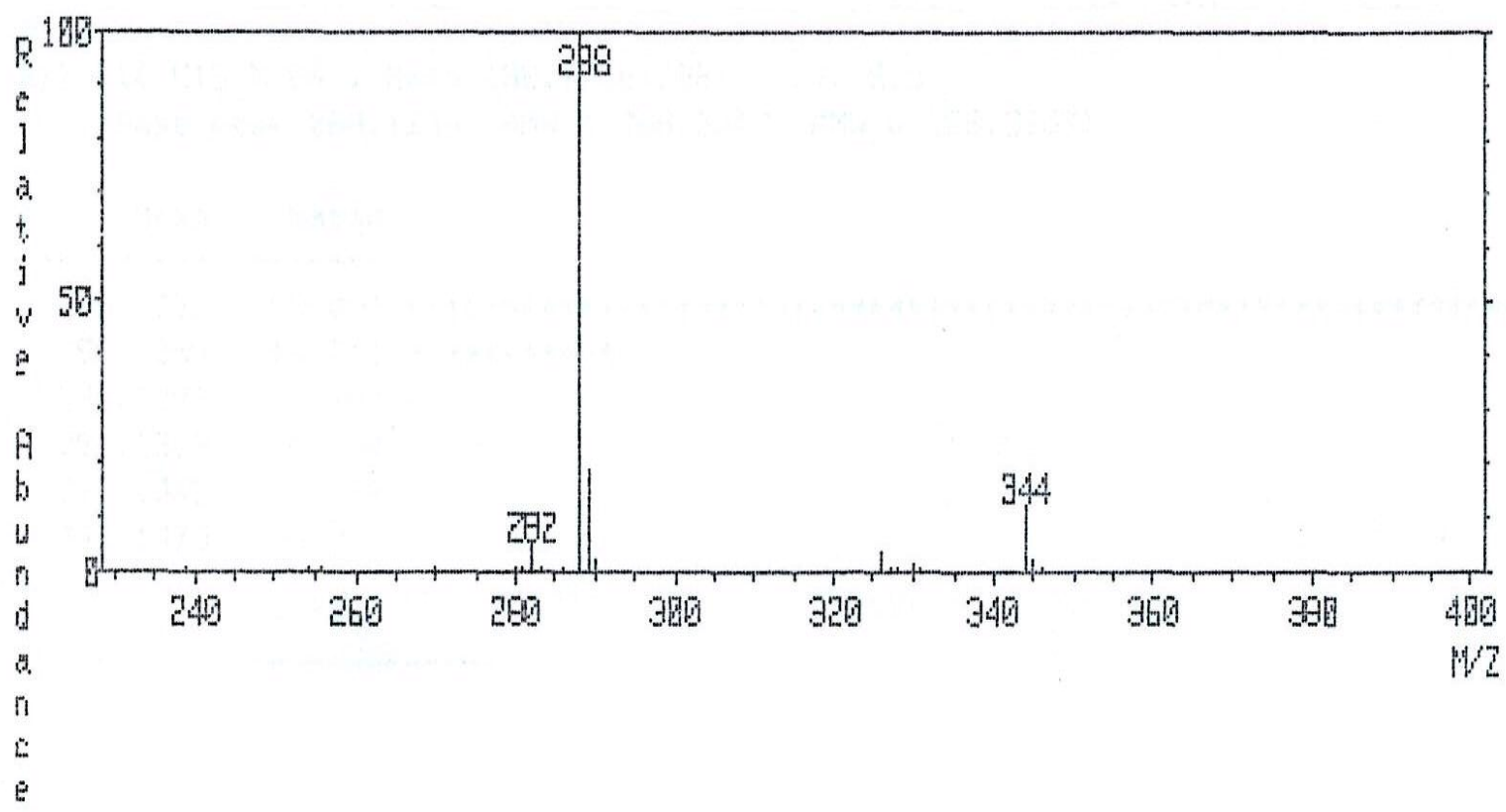



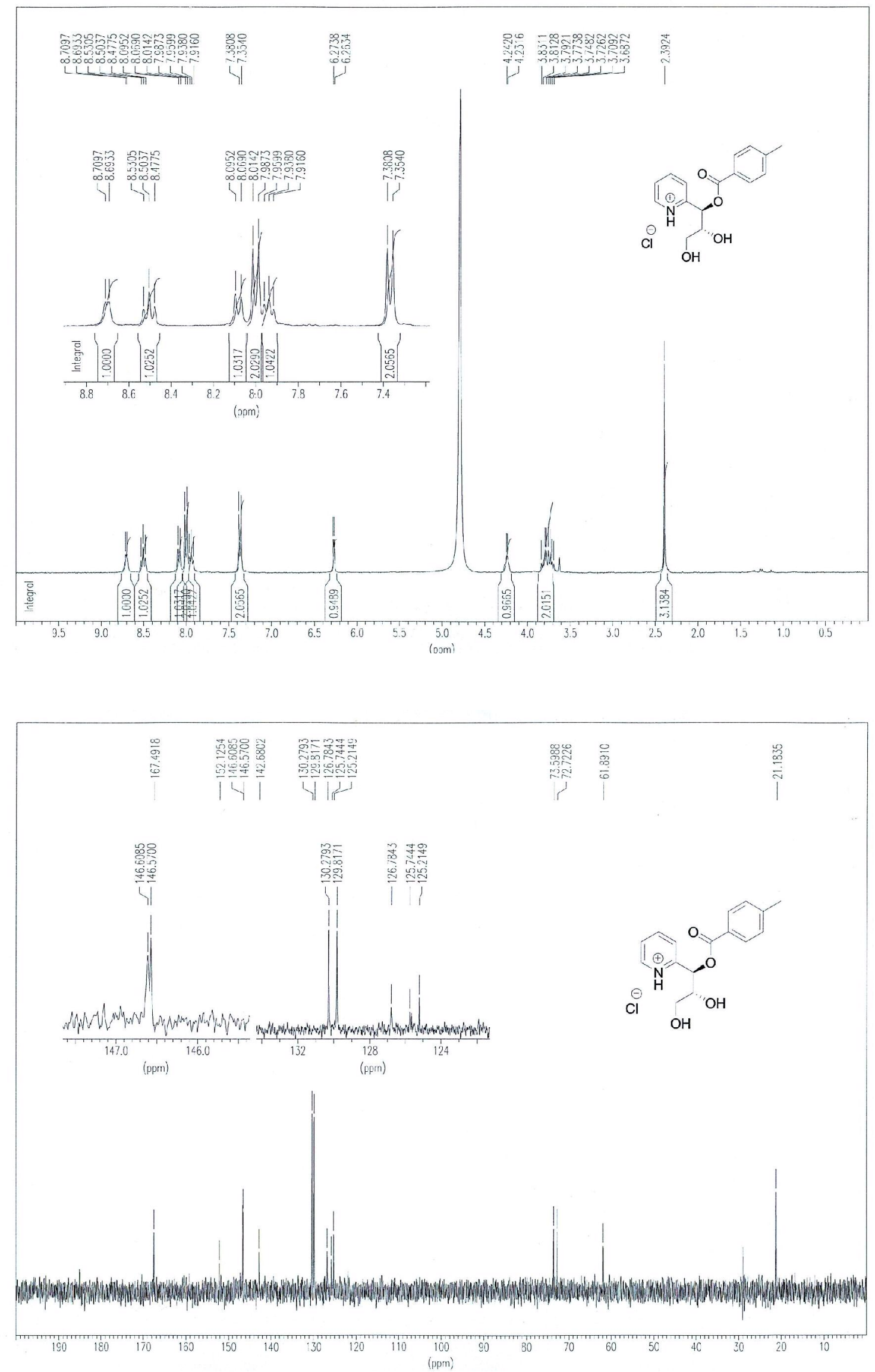

(1R,2R)-2-hydroxy-1-(4-methylbenzoyloxy)-2,3-dihydro-1H-indolizinium chloride $\mathbf{1 4}$ 


Mol. Formula : $\mathrm{C}_{16} \mathrm{H}_{16} \mathrm{ClNO}_{3}$

To a solution of $13(127 \mathrm{mg}, 0.39 \mathrm{mmol})$ and triphenylphosphine (257 $\mathrm{mg}, 0.98 \mathrm{mmol})$ in anhydrous $\mathrm{CH}_{3} \mathrm{CN}(30 \mathrm{~mL})$ was added diisopropylazodicarboxylate (DIAD) $(0.19 \mathrm{~mL}, 0.98$ mmol) dropwise at $0^{\circ} \mathrm{C}$. After stirring $2 \mathrm{~h}$ at room temperature, $\mathrm{CH}_{3} \mathrm{CN}$ was evaporated and the residue was dissolved in $\mathrm{H}_{2} \mathrm{O}$, washed with $\mathrm{Et}_{2} \mathrm{O}$ and freeze-dried to afford pure compound 14 (118 mg, $99 \%)$.

${ }^{1} \mathrm{H}$ NMR $\left(\mathrm{D}_{2} \mathrm{O}\right): 2.4(\mathrm{~s}, 3 \mathrm{H}), 3.8(\mathrm{~m}, 2 \mathrm{H}), 4.2(\mathrm{~d}, J=3.7,2 \mathrm{H}), 5.1(\mathrm{~m}, 1 \mathrm{H}), 5.3(\mathrm{dd}, J=5.9$, $13.5 \mathrm{~Hz}, 1 \mathrm{H}), 6.6(\mathrm{~d}, J=3.1,1 \mathrm{H}), 7.4(\mathrm{~d}, J=8.2,2 \mathrm{H}), 7.9(\mathrm{~d}, J=8.3,2 \mathrm{H}), 8.1(\mathrm{dd}, J=6.6,7.9$, $1 \mathrm{H}), 8.3(\mathrm{~d}, J=7.9,1 \mathrm{H}), 8.6(\mathrm{t}, J=8.9,1 \mathrm{H}), 9(\mathrm{~d}, J=6.6,1 \mathrm{H})$

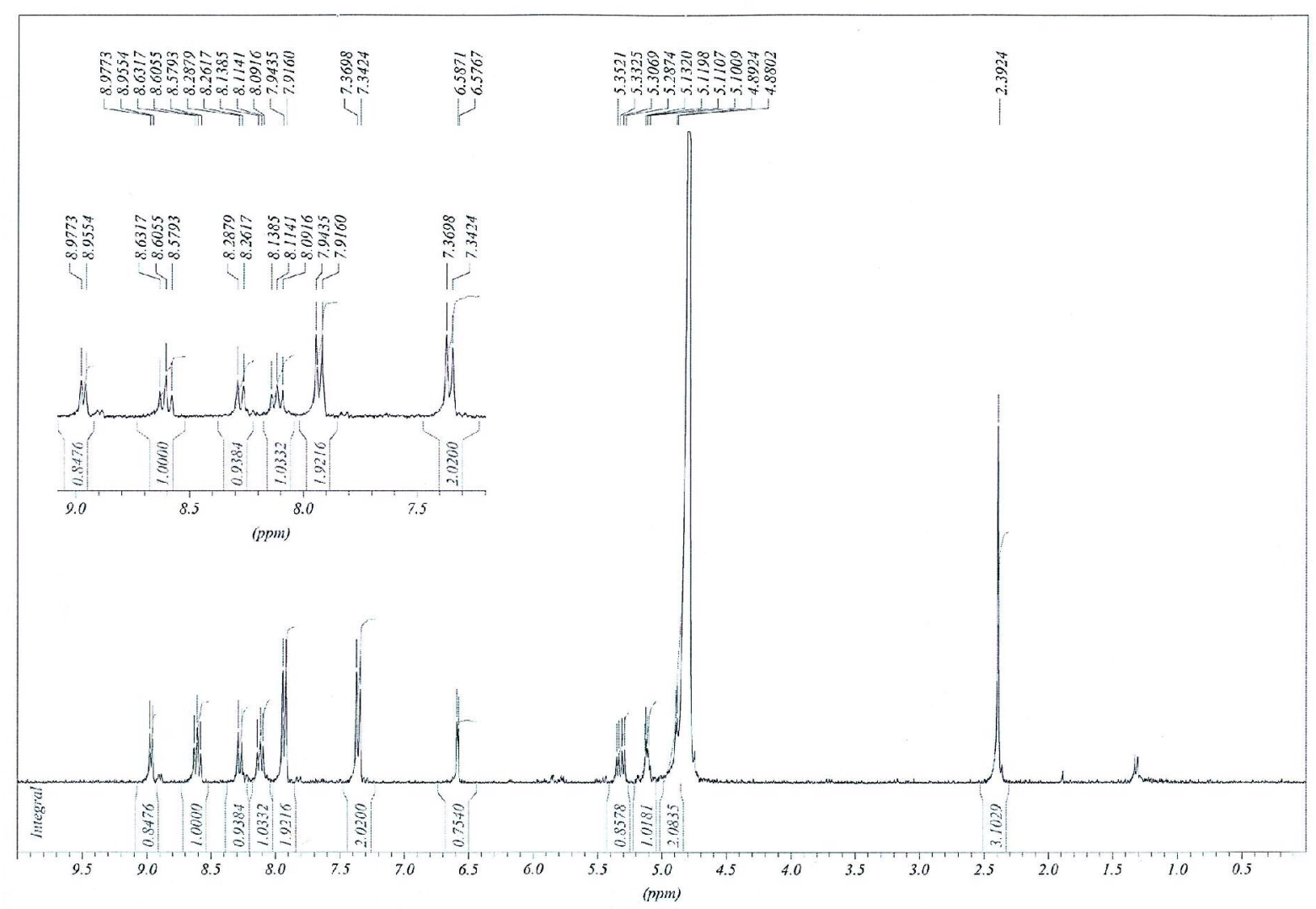

(1R,2R,8aS)-2-hydroxy-1-(4-methylbenzoyloxy)-octahydro-1H-indolizinium chloride $\mathbf{1 5}$ 


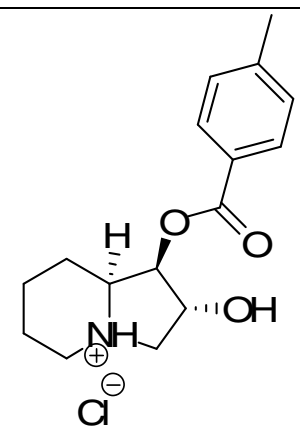

Mol. Formula : $\mathrm{C}_{16} \mathrm{H}_{22} \mathrm{ClNO}_{3}$

Mol. Weight : 311.80

A solution of compound $14(80 \mathrm{mg}, 0.26 \mathrm{mmol})$, in ethanol $(20 \mathrm{~mL})$ was stirred at room temperature in the presence of $\mathrm{PtO}_{2} \cdot \mathrm{H}_{2} \mathrm{O}(2.6 \mathrm{mg}, 10 \% \mathrm{~mol})$ under an atmospheric pressure of hydrogen. Filtration through a celite pad and evaporation in vacuo afforded compound 15 (77 $\mathrm{mg}, 95 \%$ yield) as a white powder.

${ }^{1} \mathrm{H}$ NMR $\left(\mathrm{D}_{2} \mathrm{O}\right): 1.4-2.1(\mathrm{~m},>6 \mathrm{H}$, solvent impurities $), 2.4(\mathrm{~s}, 3 \mathrm{H}), 3.1(\mathrm{~m}, 2 \mathrm{H}), 3.7(\mathrm{~m}, 2 \mathrm{H})$, $4.1(\mathrm{dd}, J=6.9,12.9,1 \mathrm{H}), 4.5(\mathrm{t}, J=12.6,1 \mathrm{H}), 5.3$ (d, $J=3.5,1 \mathrm{H}), 7.3(\mathrm{~d}, J=8,2 \mathrm{H}), 7.9$ (d, $J=8$, $2 \mathrm{H})$.

${ }^{13} \mathrm{C}$ NMR $\left(\mathrm{D}_{2} \mathrm{O}\right): 21.2,21.7,23,23.3,53.7,58.8,67.2,72.1,79.4,125.5,129.8,130.1,146.2$, 167.1

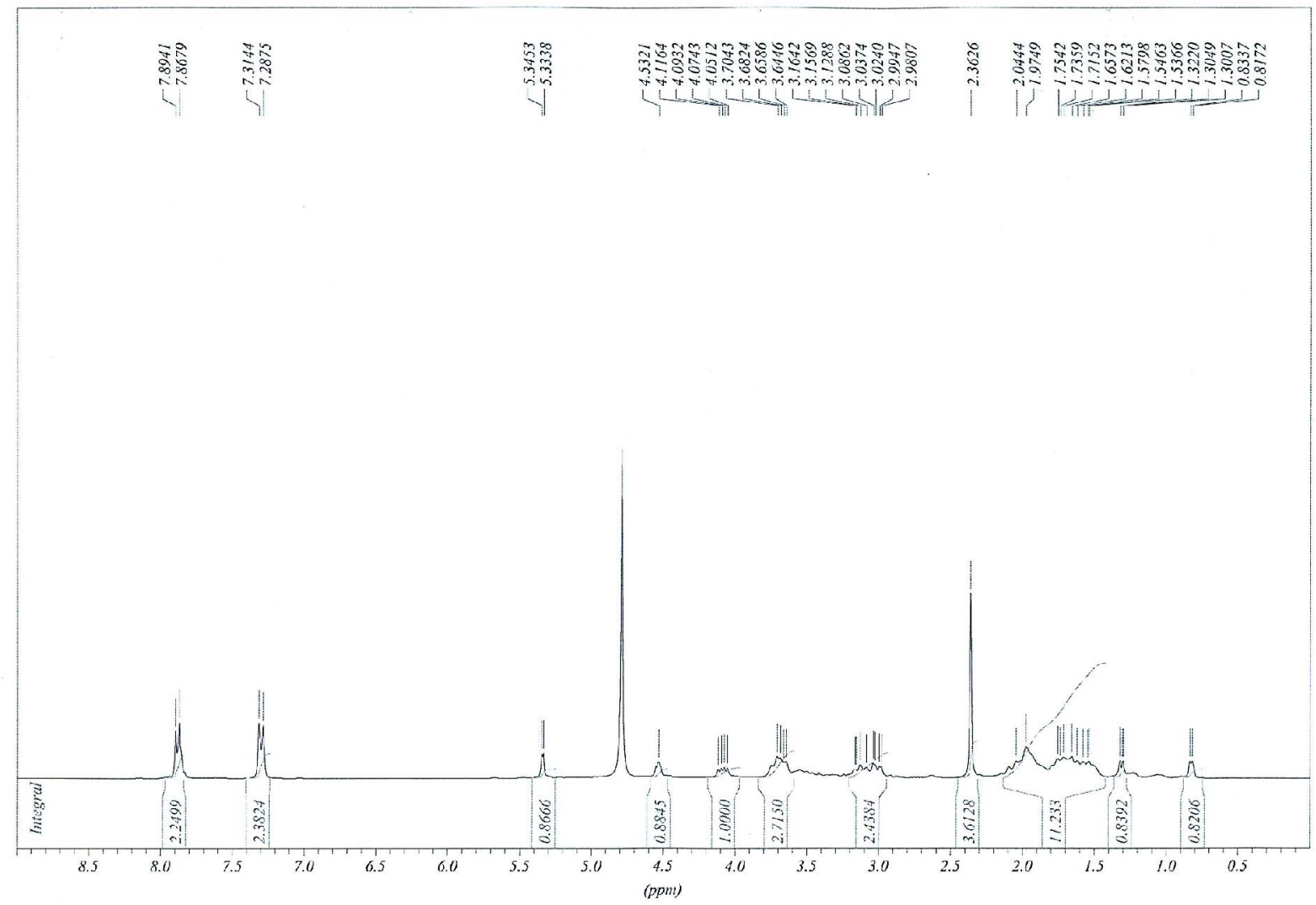




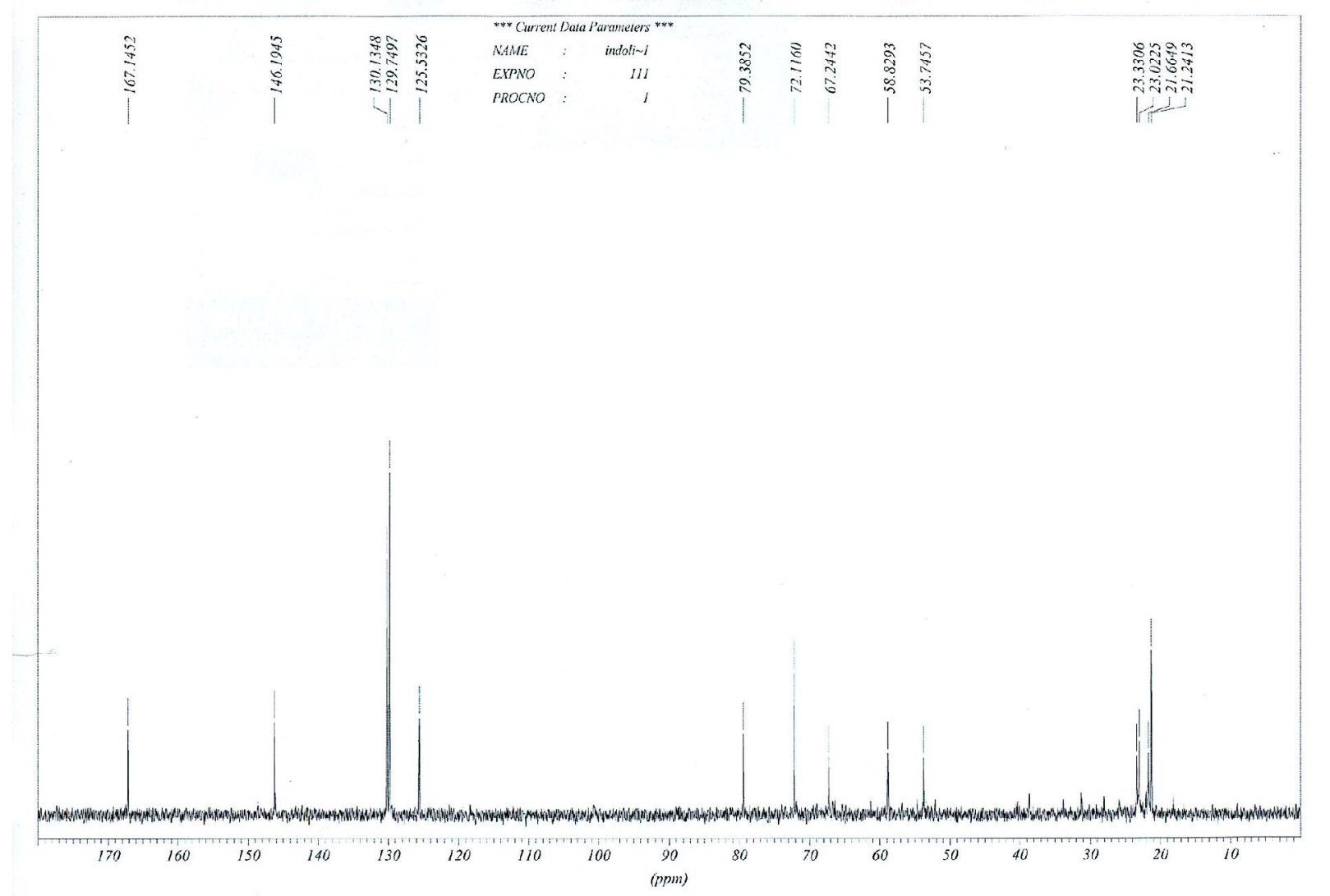

(R)-(2,2-dimethyl-1,3-dioxolan-4-yl)-(pyridine-2-yl)methanone (not numbered in the article, only provided for supplementary characterization)<smiles>CC1(C)OC[C@H](C(=O)c2ccccn2)O1</smiles>

Mol. Formula : $\mathrm{C}_{11} \mathrm{H}_{13} \mathrm{NO}_{3}$

Mol. Weight : 207.23

$n$-Butyllithium $(4.6 \mathrm{~mL}, 1.5 \mathrm{M}, 6.91 \mathrm{mmol})$ was added dropwise during $30 \mathrm{~min}$ to a solution of 2-bromopyridine $(0.6 \mathrm{~mL}, 6.28 \mathrm{mmol})$ in dry diethyl ether $(40 \mathrm{~mL})$ at $-78^{\circ} \mathrm{C}$ under nitrogen. The reaction mixture was stirred for $15 \mathrm{~min}$ and (R)-methyl-2,2-dimethyl-1,3dioxolane-4-carboxylate $(1 \mathrm{~mL}, 6.91 \mathrm{mmol})$ in diethyl ether $(5 \mathrm{~mL})$, cooled to $-78^{\circ} \mathrm{C}$, was added dropwise during $15 \mathrm{~min}$. The reaction mixture was stirred stirred at $-78^{\circ} \mathrm{C}$ for $4 \mathrm{~h}$. The reaction was quenched with saturated aqueous $\mathrm{NH}_{4} \mathrm{Cl}(30 \mathrm{~mL})$. After extractive work-up with $\mathrm{CH}_{2} \mathrm{Cl}_{2}$ and drying over $\mathrm{MgSO}_{4}$, evaporation in vacuo gave a brown oil which was purified by flash chromatography, eluting with ethyl acetate/petroleum ether : $1 / 2(\mathrm{R} f=0.5)$ to provide the ketone $(1.0 \mathrm{~g}, 88 \%)$ as a white powder $\left(\mathrm{mp}<50^{\circ} \mathrm{C}\right)$.

${ }^{1} \mathrm{H}$ NMR $\left(\mathrm{CDCl}_{3},\right): 1.49(\mathrm{~s}, 3 \mathrm{H}), 1.54(\mathrm{~s}, 3 \mathrm{H}), 4.00(\mathrm{dd}, J=8.8,6.3,1 \mathrm{H}), 4.65(\mathrm{t}, J=8.4,1 \mathrm{H})$, $5.76(\mathrm{dd}, J=8.1,6.3,1 \mathrm{H}), 7.49(\mathrm{ddd}, J=7.8,4.8,1.7,1 \mathrm{H}), 7.86(\mathrm{td}, J=7.8,0.9,1 \mathrm{H}), 8.09$ $(\mathrm{dt}, J=7.8,1.7,1 \mathrm{H}), 8.64(\mathrm{ddd}, J=4.8,1.7,0.9,1 \mathrm{H})$.

${ }^{13} \mathrm{C} \mathrm{NMR}\left(\mathrm{CDCl}_{3}\right): 25.7,26.1,67.4,78.4,110.9,122.7,127.8,137.2,149.1,151.9,197.0$ $\mathrm{IC}(+) \mathrm{MS}(\mathrm{MH}+) \mathrm{m} / \mathrm{z}=208(100 \%), 150$.

HRMS calcd for $\mathrm{C}_{11} \mathrm{H}_{14} \mathrm{NO}_{3}(\mathrm{MH}+)$ : 208.0983 found 208.0972

Anal. calcd for $\mathrm{C}_{11} \mathrm{H}_{15} \mathrm{NO}_{3}$ : C, 63.76; H, 6.32; N, 6.76 found $\mathrm{C}, 63.73 ; \mathrm{H}, 6.29 ; \mathrm{N}, 6.60$ 
SE GPECTFH Data File: GP110G7

$5+104-715=47$

Arple: ARI Ed CI $\leftarrow$ touH

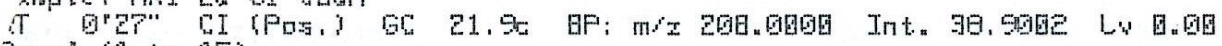

$60 \mathrm{n}$ (1) to $15 !$
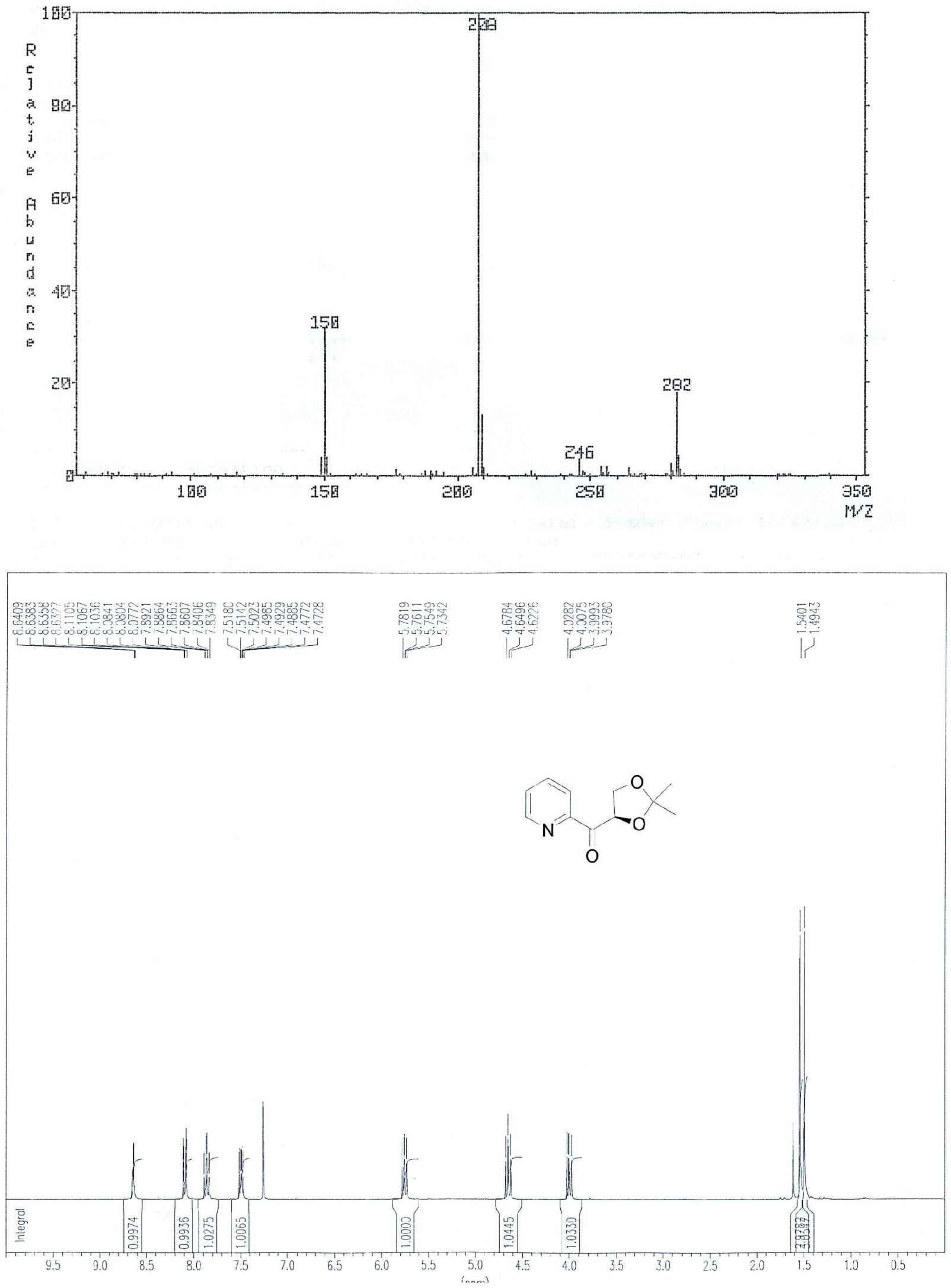


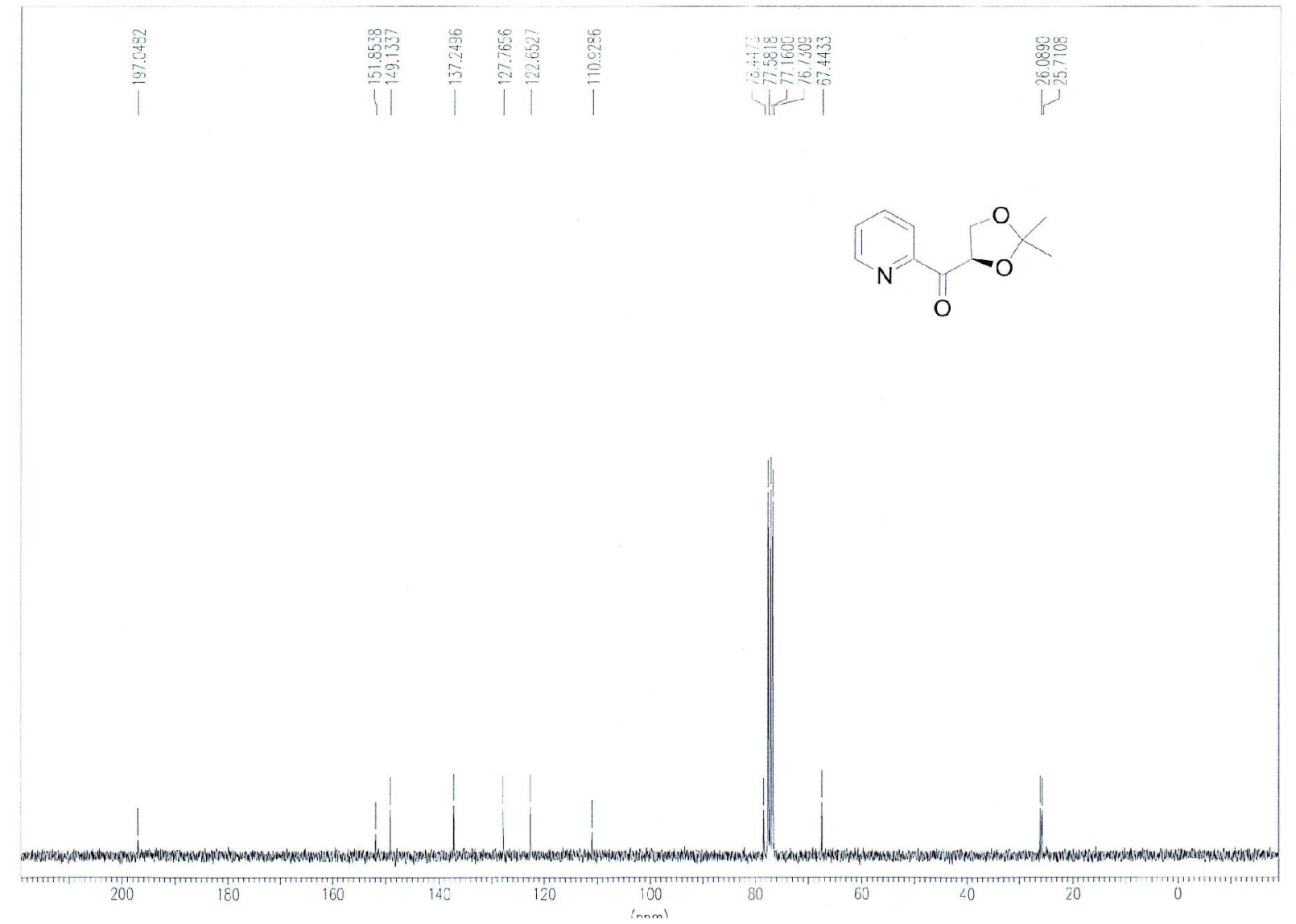

